\title{
Super Landau-Ginzburg mirrors and algebraic cycles
}

\author{
Richard S. Garavuso, ${ }^{a}$ Ludmil Katzarkov, ${ }^{b, c}$ Maximilian Kreuzer ${ }^{d}$ and Alexander Noll ${ }^{b}$ \\ ${ }^{a}$ Department of Mathematical and Statistical Sciences, University of Alberta \\ 632 Central Academic Building; Edmonton, Alberta T6G 2G1; Canada \\ ${ }^{b}$ Department of Mathematics, Universität Wien \\ Garnisongasse 3, Vienna A-1090; Austria \\ ${ }^{c}$ Department of Mathematics, University of Miami \\ PO Box 249085; Coral Gables, FL 33124-4250; United States \\ ${ }^{d}$ Institut für Theoretische Physik, Technische Universität Wien \\ Wiedner Hauptstrasse 8-10/136; Vienna A-1040; Austria \\ E-mail: garavuso@ualberta.ca, lkatzark@math.uci.edu, \\ nolla5@univie.ac.at
}

ABSTRACT: We investigate the super Landau-Ginzburg mirrors of gauged linear sigma models which, in an appropriate low energy limit, reduce to nonlinear sigma models with Kähler supermanifold target spaces of nonnegative super-first Chern class.

Keywords: Conformal Field Models in String Theory, Sigma Models, String Duality

Dedicated to our dear friend and coauthor Maximilian Kreuzer, who died November 26, 2010. 


\section{Contents}

1 Introduction 1

$\mathbf{2} \mathbf{W C P}^{m-1 \mid n} \quad \mathbf{3}$

$\mathbf{3} \operatorname{Tot}\left(\mathcal{O}(-s) \rightarrow \mathbf{W C P}^{m-1 \mid n}\right) \quad \mathbf{4}$

4 Hypersurface in $\mathbf{W C P}^{m-1 \mid n} \quad \mathbf{5}$

5 Complete intersection in $\mathbf{W C P}^{m-1 \mid n} \quad 11$

6 Complete intersection in a general toric supervariety 12

$\begin{array}{lll}7 & \text { Period relations } & 15\end{array}$

$\begin{array}{lll}8 & \text { Categorical interpretation } & 17\end{array}$

$\begin{array}{ll}\text { 8.1 Supermanifold cohomology calculations } & 17\end{array}$

$\begin{array}{ll}\text { 8.2 Super and DG scheme version of Homological Mirror Symmetry } & 18\end{array}$

$\begin{array}{ll}\text { 8.3 Algebro-geometric applications } & 19\end{array}$

8.4 Gaps and spectra 21

$\begin{array}{lll}\text { 8.5 Supermanifolds and exotic }(p, p) \text { cycles } & 22\end{array}$

A A-model super CY hypersurface $\Leftrightarrow$ B-model quasihomogeneity $\quad 25$

\section{Introduction}

A Kähler manifold $M$ with nonnegative first Chern class can be described in terms of a $(2,2)$ supersymmetric gauged linear sigma model in $1+1$ dimensions $[1,2]$. That is, in an appropriate low energy limit, the gauge theory reduces to a nonlinear sigma model with resolved target space $M$. Hori and Vafa [3] proved that the gauged linear sigma model corresponding to $M$ is mirror to a Landau-Ginzburg theory. When $M$ is a CalabiYau manifold, the mirror Landau-Ginzburg theory can sometimes be given a geometrical interpretation in terms of a nonlinear sigma model with Calabi-Yau resolved target space $\widetilde{M}$. Here, the manifold $\widetilde{M}$ is the mirror of $M$.

A rigid Calabi-Yau manifold has no complex structure moduli. The mirror of such a manifold has no Kähler moduli and hence cannot be a Kähler manifold in the conventional sense. Thus, Kähler manifolds cannot be the most general geometrical framework for understanding mirror symmetry. The first progress towards generalizing this framework came when it was suggested that higher-dimensional Fano varieties could provide the mirrors for rigid Calabi-Yau manifolds $[4,5]$. Later progress came when Sethi [6] proposed a general 
correspondence between orbifolds of $(2,2)$ supersymmetric Landau-Ginzburg theories in $1+1$ dimensions with integral $\hat{c} \equiv c / 3$ (where $c$ is the central charge) and nonlinear sigma models. Here, the resolved target space of the nonlinear sigma model is either a Calabi-Yau manifold or a Calabi-Yau supermanifold. Using this proposal, Sethi argued that the mirror of a rigid Calabi-Yau manifold is a Calabi-Yau supermanifold and that supervarieties are the proper geometrical framework of mirror symmetry.

In the absence of a proper supercohomology theory, Sethi used heuristic arguments for computing the Hodge numbers of Calabi-Yau supermanifolds realized (at least in principle) as crepant resolutions of hypersurfaces in weighted complex superprojective spaces. Using these same heuristic arguments, the geometrical interpretations associated with Sethi's proposed correspondence were investigated in [7]. As might be expected, it was found that the supermanifold Hodge numbers obtained by using these heuristic arguments do not always agree with those of the corresponding Landau-Ginzburg orbifold.

Aganagic and Vafa [8] showed that when $M$ is a Calabi-Yau supermanifold realized as a crepant resolution of a weighted complex superprojective space, the corresponding gauged linear sigma model is mirror to a super Landau-Ginzburg theory. This relation should also hold when $M$ is any Kähler supermanifold with nonnegative super-first Chern class. In the super Calabi-Yau case, one expects to obtain a geometrical intepretation of the super Landau-Ginzburg mirror, i.e. a nonlinear sigma model with super Calabi-Yau resolved target space $\widetilde{M}$. Various examples of such geometrical interpretations are given in $[8-12]$.

In this paper, we will discuss the super Landau-Ginzburg mirrors of gauged linear sigma models corresponding to Kähler supermanifolds with nonnegative super-first Chern class. In Sections 2, 3, 4, 5, and 6, respectively, we will consider the cases in which the Kähler supermanifold is realized as a crepant resolution of

(i) a weighted complex superprojective space $\mathbf{W} \mathbf{C P}^{m-1 \mid n}$,

(ii) $\operatorname{Tot}\left(\mathcal{O}(-s) \rightarrow \mathbf{W C P}^{m-1 \mid n}\right)$,

(iii) a hypersurface in $\mathbf{W C} \mathbf{P}^{m-1 \mid n}$,

(iv) a complete intersection in $\mathbf{W C P} \mathbf{P}^{m-1 \mid n}$, and

(v) a complete intersection in a general toric supervariety.

For cases (i) - (iv), the unresolved variety corresponds to a gauged linear sigma model with $U(1)$ gauge group. For case (iii), when the super Calabi-Yau condition is satisfied, we will give a geometrical interpretation of the super Landau-Ginzburg mirror. Our analysis here will include two examples which can be used to test Sethi's proposed correspondence using the recently developed techniques of [13]. For case (v), we will show that the mirror theory can be expressed as a super Landau-Ginzburg theory on a noncompact supermanifold. In Section 7, we will establish some relations between periods of mirrors of gauged linear sigma models corresponding to various geometries. These relations generalize results which were obtained in [14] by working with A-models. In Section 8, we will give a categorical interpre- 
tation of the material presented in Sections 2 - 7. Finally, in the Appendix, we will prove a theorem and corollary concerning the quasihomogeneity of the mirror superpotential for case (iii).

\section{$2 \mathbf{W C P}^{m-1 \mid n}$}

Consider a weighted complex superprojective space $\mathbf{W C P}_{\left(Q_{1}, \ldots, Q_{m} \mid q_{1}, \ldots, q_{n}\right)}^{m-1 \mid n}$ with $m$ homogeneous bosonic coordinates $\phi_{i}$ having weights $Q_{i}$, where $i=1, \ldots, m$, and $n$ homogeneous fermionic coordinates $\xi_{a}$ having weights $q_{a}$, where $a=1, \ldots, n$, i.e.

$$
\left(\phi_{1}, \ldots, \phi_{m} \mid \xi_{1}, \ldots, \xi_{n}\right) \simeq\left(\lambda^{Q_{1}} \phi_{1}, \ldots, \lambda^{Q_{m}} \phi_{m} \mid \lambda^{q_{1}} \xi_{1}, \ldots, \lambda^{q_{n}} \xi_{n}\right), \quad \lambda \in \mathbf{C}^{*} .
$$

A Kähler supermanifold may be obtained as a crepant resolution of this supervariety if a crepant resolution exists. Such a supermanifold would have nonnegative super-first Chern class when

$$
\sum_{i=1}^{m} Q_{i}-\sum_{a=1}^{n} q_{a} \geq 0
$$

We will assume that a crepant resolution exists, the condition (2.1) is satisfied, and the bosonic and fermionic weights are positive integers. The Kähler supermanifold that we obtain can be described as the resolved target space of a nonlinear sigma model phase of a $(2,2)$ supersymmetric $U(1)$ gauged linear sigma model with $m$ bosonic chiral superfields $\Phi_{i}$ having $U(1)$ charges $Q_{i}, i=1, \ldots, m$, and $n$ fermionic chiral superfields $\Xi_{a}$ having $U(1)$ charges $q_{a}, a=1, \ldots, n$. The classical Lagrangian is [15]

$$
\begin{aligned}
L_{\mathbf{W C P}_{\left(Q_{1}, \ldots, Q_{m} \mid q_{1}, \ldots, q_{n}\right)}^{m-1 \mid n}} & =\int d^{4} \theta\left(\sum_{i=1}^{m} \bar{\Phi}_{i} e^{2 Q_{i} V} \Phi_{i}+\sum_{a=1}^{n} \bar{\Xi}_{a} e^{2 q_{a} V} \Xi_{a}-\frac{1}{2 e^{2}} \bar{\Sigma} \Sigma\right) \\
& -\frac{1}{2}\left(t \int d^{2} \tilde{\theta} \Sigma+c . c .\right),
\end{aligned}
$$

where $\Sigma=\bar{D}_{+} D_{-} V$ is the twisted chiral field strength of the $U(1)$ vector superfield $V, e$ is the $U(1)$ gauge coupling, and $t=r-i \vartheta$ is the complexified Fayet-Iliopoulos parameter. The nonlinear sigma model phase is realized in the low energy limit with $r>>0$ with $\sigma=0$ and target space

$$
\frac{\left\{\left.\left(\phi_{1}, \ldots, \phi_{m} \mid \xi_{1}, \ldots, \xi_{n}\right)\left|\sum_{i=1}^{m} Q_{i}\right| \phi_{i}\right|^{2}+\sum_{a=1}^{n} q_{a}\left|\xi_{a}\right|^{2}=r\right\}}{U(1)} .
$$

Here, $\sigma, \phi_{i}$ and $\xi_{a}$ are respectively the lowest components of $\Sigma, \Phi_{i}$, and $\Xi_{a}$.

The gauge theory is super-renormalizable with respect to $e$. When the super CalabiYau condition

$$
\sum_{i=1}^{m} Q_{i}-\sum_{a=1}^{n} q_{a}=0
$$

is not satisfied, to cancel a one-loop ultraviolet divergence, the Fayet-Iliopoulos parameter $r$ must be renormalized as

$$
r(\mu)=r\left(\Lambda_{U V}\right)+\left(\sum_{i=1}^{m} Q_{i}-\sum_{a=1}^{n} q_{a}\right) \ln \left(\frac{\mu}{\Lambda_{U V}}\right),
$$


where $r(\mu)$ is the renormalized Fayet-Iliopoulos parameter at the scale $\mu, \Lambda_{U V}$ is the ultraviolet cutoff, and

$$
r\left(\Lambda_{U V}\right)=\left(\sum_{i=1}^{m} Q_{i}-\sum_{a=1}^{n} q_{a}\right) \ln \left(\frac{\Lambda_{U V}}{\Lambda}\right) .
$$

In this case, the dimensionless parameter $r$ of the classical theory is replaced by the renormalization group invariant dynamical scale $\Lambda$ in the quantum theory.

The classical theory possesses a $U(1)_{V}$ vector R-symmetry when $\Sigma$ is assigned vector charge 0 . It also has a $U(1)_{A}$ axial R-symmetry when $\Sigma$ is assigned axial charge 2 . The vector R-symmetry is an exact symmetry of the quantum theory, but the axial R-symmetry is subject to a chiral anomaly. An axial rotation by angle $\alpha$ shifts the theta angle as

$$
\vartheta \rightarrow \vartheta-2\left(\sum_{i=1}^{m} Q_{i}-\sum_{a=1}^{n} q_{a}\right) \alpha .
$$

Note that the axial anomaly vanishes when the super Calabi-Yau condition (2.4) is satisfied.

Following the arguments of [8], we obtain the super Landau-Ginzburg mirror period

$$
\begin{aligned}
\Pi_{\widetilde{\mathbf{W C P}}_{\left(Q_{1}, \ldots, Q_{m} \mid q_{1}, \ldots, q_{n}\right)}^{m-1 \mid n}} & \left(\prod_{i=1}^{m} d Y_{i}\right)\left(\prod_{a=1}^{n} d X_{a} d \eta_{a} d \gamma_{a}\right) \delta\left(\sum_{i=1}^{m} Q_{i} Y_{i}-\sum_{a=1}^{n} q_{a} X_{a}-t\right) \\
= & \quad \times \exp \left[-\sum_{i=1}^{m} e^{-Y_{i}}-\sum_{a=1}^{n} e^{-X_{a}}\left(1+\eta_{a} \gamma_{a}\right)\right],
\end{aligned}
$$

where $Y_{i}$ and $X_{a}$ are twisted chiral superfields (with periodicity $2 \pi i$ ) which satisfy

$$
\operatorname{Re} Y_{i}=\bar{\Phi}_{i} e^{2 Q_{i} V} \Phi_{i}, \quad \operatorname{Re} X_{a}=-\bar{\Xi}_{a} e^{2 q_{a} V} \Xi_{a}
$$

and $\eta_{a}$ and $\gamma_{a}$ are fermionic superfields. In fact, (2.5) holds whenever (2.1) is satisfied.

$$
3 \operatorname{Tot}\left(\mathcal{O}(-s) \rightarrow \mathbf{W C P}^{m-1 \mid n}\right)
$$

Consider the noncompact supervariety $\operatorname{Tot}\left(\mathcal{O}(-s) \rightarrow \mathbf{W C P}_{\left(Q_{1}, \ldots, Q_{m} \mid q_{1}, \ldots, q_{n}\right)}^{m-1 \mid n}\right)$, i.e. the total space of the line bundle $\mathcal{O}(-s)$ over the weighted complex superprojective space of Section 2. A Kähler supermanifold may be obtained as a crepant resolution of this supervariety if a crepant resolution exists. Such a supermanifold would have nonnegative super-first Chern class when

$$
\sum_{i=1}^{m} Q_{i}-\sum_{a=1}^{n} q_{a}-s \geq 0 .
$$

We will assume that a crepant resolution exists, the condition (3.1) is satisfied, and $s$ is a positive integer. To describe our Kähler supermanifold in terms of a gauged linear sigma 
model, we add to (2.2) a kinetic term for a bosonic chiral superfield $P$ of $U(1)$ charge $-s$. The resulting Lagrangian is

$$
\begin{aligned}
L_{\operatorname{Tot}}\left(\mathcal{O}(-s) \rightarrow \mathbf{W C P}_{\left(Q_{1}, \ldots, Q_{m} \mid q_{1}, \ldots, q_{n}\right)}^{m-1 \mid n}\right) & \\
= & \int d^{4} \theta\left(\sum_{i=1}^{m} \bar{\Phi}_{i} e^{2 Q_{i} V} \Phi_{i}+\bar{P} e^{-2 s V} P+\sum_{a=1}^{n} \bar{\Xi}_{a} e^{2 q_{a} V} \Xi_{a}-\frac{1}{2 e^{2}} \bar{\Sigma} \Sigma\right) \\
& -\frac{1}{2}\left(t \int d^{2} \tilde{\theta} \Sigma+\text { c.c. }\right) .
\end{aligned}
$$

The nonlinear sigma model phase is realized in the low energy limit with $r \gg 0, \sigma=0$, and target space

$$
\frac{\left\{\left.\left(\phi_{1}, \ldots, \phi_{m}, p \mid \xi_{1}, \ldots, \xi_{n}\right)\left|\sum_{i=1}^{m} Q_{i}\right| \phi_{i}\right|^{2}-s|p|^{2}+\sum_{a=1}^{n} q_{a}\left|\xi_{a}\right|^{2}=r\right\}}{U(1)},
$$

where $p$ is the lowest component of $P$. If the super Calabi-Yau condition

$$
\sum_{i=1}^{m} Q_{i}-\sum_{a=1}^{n} q_{a}=s
$$

is satisfied, then the Fayet-Iliopoulos parameter $r$ does not renormalize.

Extending the result (2.5) to the present case, we obtain for the super Landau-Ginzburg mirror period

$$
\begin{aligned}
\left.\Pi_{\widetilde{\operatorname{Tot}}(\mathcal{O}(-s)} \rightarrow \mathbf{W C P}_{\left(Q_{1}, \ldots, Q_{m} \mid q_{1}, \ldots, q_{n}\right)}^{m-1 \mid}\right) & \\
=\int & \left(\prod_{i=1}^{m} d Y_{i}\right) d Y_{P}\left(\prod_{a=1}^{n} d X_{a} d \eta_{a} d \gamma_{a}\right) \delta\left(\sum_{i=1}^{m} Q_{i} Y_{i}-s Y_{P}-\sum_{a=1}^{n} q_{a} X_{a}-t\right) \\
& \times \exp \left[-\sum_{i=1}^{m} e^{-Y_{i}}-e^{-Y_{P}}-\sum_{a=1}^{n} e^{-X_{a}}\left(1+\eta_{a} \gamma_{a}\right)\right],
\end{aligned}
$$

where $Y_{P}$ is a bosonic twisted chiral superfield (with periodicity $2 \pi i$ ) which satisfies

$$
\operatorname{Re} Y_{P}=\bar{P} e^{-2 s V} P \text {. }
$$

This result will be useful in the next section where we study an associated compact theory.

\section{Hypersurface in $\mathrm{WCP}^{m-1 \mid n}$}

Consider a member of the family of compact supervarieties $\mathbf{W C P}_{\left(Q_{1}, \ldots, Q_{m} \mid q_{1}, \ldots, q_{n}\right)[s]}^{m-1 \mid n}$, i.e. a hypersurface defined by the zero locus of a quasihomogeneous polynomial $G=G(\phi, \xi)$ of degree $s$ in the weighted complex superprojective space of Section 2. A Kähler supermanifold may be obtained as a crepant resolution of this supervariety if a crepant resolution exists. Such a supermanifold would have nonnegative super-first Chern class when

$$
\sum_{i=1}^{m} Q_{i}-\sum_{a=1}^{n} q_{a}-s \geq 0
$$


We will assume that a crepant resolution exists and the condition (4.1) is satisfied. To describe our Kähler supermanifold in terms of a gauged linear sigma model, we add to (3.2) an untwisted F-term with superpotential of the form $P \cdot G(\Phi, \Xi)$, where $G(\Phi, \Xi)$ is a quasihomogeneous polynomial of $U(1)$ charge $s$. The resulting Lagrangian is

$$
\begin{aligned}
L_{\mathbf{W C P}_{\left(Q_{1}, \ldots, Q_{m} \mid q_{1}, \ldots, q_{n}\right)[s]}^{m-1 \mid n}} & =\int d^{4} \theta\left(\sum_{i=1}^{m} \bar{\Phi}_{i} e^{2 Q_{i} V} \Phi_{i}+\sum_{a=1}^{n} \bar{\Xi}_{a} e^{2 q_{a} V} \Xi_{a}+\bar{P} e^{-2 s V} P-\frac{1}{2 e^{2}} \bar{\Sigma} \Sigma\right) \\
& -\frac{1}{2}\left(t \int d^{2} \tilde{\theta} \Sigma+\text { c.c. }\right)+\left(\int d^{2} \theta P \cdot G(\Phi, \Xi)+\text { c.c. }\right) .
\end{aligned}
$$

The nonlinear sigma model phase is realized in the low energy limit with $r \gg 0, \sigma=0$, $p=0$, and target space

$$
\{G=0\} \subset \frac{\left\{\left.\left(\phi_{1}, \ldots, \phi_{m} \mid \xi_{1}, \ldots, \xi_{n}\right)\left|\sum_{i=1}^{m} Q_{i}\right| \phi_{i}\right|^{2}+\sum_{a=1}^{n} q_{a}\left|\xi_{a}\right|^{2}=r\right\}}{U(1)} .
$$

If the super Calabi-Yau condition

$$
\sum_{i=1}^{m} Q_{i}-\sum_{a=1}^{n} q_{a}=s
$$

is satisfied, then the Fayet-Iliopoulos parameter $r$ does not renormalize.

Generalizing the discussion in [3], we can obtain the super Landau-Ginzburg mirror period by allowing the operator $-s \partial / \partial t$ to act on the period given by (3.5), i.e.

$$
\begin{aligned}
\Pi_{\widetilde{\mathbf{W C P}}_{\left(Q_{1}, \ldots, Q_{m} \mid q_{1}, \ldots, q_{n}\right)[s]}^{m-1 \mid n}} & \\
=- & s \frac{\partial}{\partial t} \Pi_{\widehat{\operatorname{Tot}}\left(\mathcal{O}(-s) \rightarrow \mathbf{W C P}_{\left(Q_{1}, \ldots, Q_{m} \mid q_{1}, \ldots, q_{n}\right)}^{m-1 \mid n}\right)} \\
= & \int\left(\prod_{i=1}^{m} d Y_{i}\right) d Y_{P} e^{-Y_{P}}\left(\prod_{a=1}^{n} d X_{a} d \eta_{a} d \gamma_{a}\right) \delta\left(\sum_{i=1}^{m} Q_{i} Y_{i}-s Y_{P}-\sum_{a=1}^{n} q_{a} X_{a}-t\right) \\
& \quad \times \exp \left[-\sum_{i=1}^{m} e^{-Y_{i}}-e^{-Y_{P}}-\sum_{a=1}^{n} e^{-X_{a}}\left(1+\eta_{a} \gamma_{a}\right)\right] .
\end{aligned}
$$

Integrating (4.5) over $Y_{P}$ yields

$$
\begin{aligned}
& \Pi_{\widetilde{\mathbf{W C P}}_{\left(Q_{1}, \ldots, Q_{m} \mid q_{1}, \ldots, q_{n}\right)[s]}^{m-1 \mid s}}\left(\prod_{i=1}^{m} d Y_{i}\right)\left[e^{\frac{t}{s}} \prod_{i=1}^{m}\left(e^{-Y_{i}}\right)^{\frac{Q_{i}}{s}} \prod_{a=1}^{n}\left(e^{-X_{a}}\right)^{-\frac{q_{a}}{s}}\right]\left(\prod_{a=1}^{n} d X_{a} d \eta_{a} d \gamma_{a}\right) \\
& \quad \times \exp \left[-\sum_{i=1}^{m} e^{-Y_{i}}-e^{\frac{t}{s}} \prod_{i=1}^{m}\left(e^{-Y_{i}}\right)^{\frac{Q_{i}}{s}} \prod_{a=1}^{n}\left(e^{-X_{a}}\right)^{-\frac{q_{a}}{s}}-\sum_{a=1}^{n} e^{-X_{a}}\left(1+\eta_{a} \gamma_{a}\right)\right] .
\end{aligned}
$$


Suppose there exist invertible matrices $\left(M_{j i}\right)$ and $\left(N_{b a}\right)$ of nonnegative integers such that

$$
s=\sum_{i=1}^{m} M_{j i} Q_{i}=\sum_{a=1}^{n} N_{b a} q_{a}, \quad j=1, \ldots, m ; \quad b=1, \ldots, n .
$$

Now, consider the change of variables

$$
e^{-Y_{i}}=\prod_{j=1}^{m} y_{j}^{M_{j i}}, \quad e^{-X_{a}}=\prod_{b=1}^{n} x_{b}^{N_{b a}}, \quad \eta_{a}=x_{a}^{-1} \hat{\eta}_{a}, \quad \gamma_{a}=x_{a}^{-1} \hat{\gamma}_{a} .
$$

This change of variables one-to-one up to the action of the group $\Gamma$ defined by

$$
\Gamma: y_{j} \rightarrow \omega_{y_{j}} y_{j}, \quad x_{b} \rightarrow \omega_{x_{b}} x_{b}, \quad \hat{\eta}_{a} \rightarrow \omega_{x_{a}} \hat{\eta}_{a}, \quad \hat{\gamma}_{a} \rightarrow \omega_{x_{a}} \hat{\gamma}_{a},
$$

such that

$$
\prod_{j=1}^{m} \omega_{y_{j}}^{M_{j i}}=1, \quad \prod_{b=1}^{n} \omega_{x_{b}}^{N_{b a}}=1, \quad \prod_{j=1}^{m} \omega_{y_{j}} \prod_{b=1}^{n} \omega_{x_{b}}^{-1}=1 .
$$

In terms of the new variables, we obtain

$$
\begin{aligned}
& \Pi_{\widetilde{\mathbf{W C P}}_{\left(Q_{1}, \ldots, Q_{m} \mid q_{1}, \ldots, q_{n}\right)[s]}^{m-1 \mid n}} \\
& =(-1)^{m+n} \operatorname{det}\left(M_{j i}\right) \operatorname{det}\left(N_{b a}\right) e^{t / s} \int\left(\prod_{i=1}^{m} d y_{i}\right)\left(\prod_{a=1}^{n} d x_{a} d \hat{\eta}_{a} d \hat{\gamma}_{a}\right) \\
& \quad \times \exp \left[-\sum_{i=1}^{m} \prod_{j=1}^{m} y_{j}^{M_{j i}}-e^{t / s} \prod_{j=1}^{m} y_{j} \prod_{b=1}^{n} x_{b}^{-1}-\sum_{a=1}^{n}\left(1+x_{a}^{-2} \hat{\eta}_{a} \hat{\gamma}_{a}\right) \prod_{b=1}^{n} x_{b}^{N_{b a}}\right] .
\end{aligned}
$$

This is the period for the super Landau-Ginzburg orbifold

$$
\widetilde{W} / \Gamma
$$

where

$$
\widetilde{W}=\sum_{i=1}^{m} \prod_{j=1}^{m} y_{j}^{M_{j i}}+e^{t / s} \prod_{j=1}^{m} y_{j} \prod_{b=1}^{n} x_{b}^{-1}+\sum_{a=1}^{n}\left(1+x_{a}^{-2} \hat{\eta}_{a} \hat{\gamma}_{a}\right) \prod_{b=1}^{n} x_{b}^{N_{b a}}
$$

and $\Gamma$ is given by (4.8).

As proven in the Appendix, $\widetilde{W}$ is quasihomogeneous of some degree $s^{\prime}$ for all values of $t$ if and only if the super Calabi-Yau condition (4.4) is satisfied. In this case, for appropriately chosen fermionic weights, the super Landau-Ginzburg orbifold $\widetilde{W} / \Gamma$ corresponds to a Calabi-Yau supermanifold obtained as a crepant resolution of

$$
\frac{\{\widetilde{W}=0\}}{\Gamma / \widetilde{J}} \subset \mathbf{W C P}_{\left(n_{y_{1}}, \ldots, n_{y_{m}}, n_{x_{1}}, \ldots, n_{x_{n}} \mid n_{\hat{\eta}_{1}}, n_{\hat{\gamma}_{1}}, \ldots, n_{\hat{\eta}_{n}}, n_{\hat{\gamma}_{n}}\right)}^{m+n-1 \mid 2 n},
$$

where $\widetilde{J}$ is the diagonal subgroup of the phase symmetries of $\widetilde{W}$, i.e.

$$
\widetilde{J}: \quad y_{j} \rightarrow e^{2 \pi i n_{y_{j}} / s^{\prime}} y_{j}, \quad x_{b} \rightarrow e^{2 \pi i n_{x_{b}} / s^{\prime}} x_{b}, \quad \hat{\eta}_{b} \rightarrow e^{2 \pi i n_{\hat{\eta}_{b}} / s^{\prime}} \hat{\eta}_{b}, \quad \hat{\gamma}_{b} \rightarrow e^{2 \pi i n_{\hat{\gamma}_{b}} / s^{\prime}} \hat{\gamma}_{b} .
$$


Example 4.1. Consider the Landau-Ginzburg orbifold $G_{b o s} / J_{b o s}$, where

$$
G_{b o s}=\phi_{1}^{6}+\phi_{2}^{6}+\phi_{3}^{6}+\phi_{4}^{3}+\phi_{5}^{3}+\phi_{6}^{3}+\phi_{7}^{2}
$$

and $J_{b o s}$ is the diagonal subgroup of the phase symmetries of $G_{b o s}$, i.e.

$$
J_{b o s}: \phi_{i} \rightarrow e^{2 \pi i Q_{i} / 6} \phi_{i} .
$$

\begin{tabular}{|c|c|c|c|}
\hline & & & \\
\hline & 0 & 0 & \\
\hline 0 & & ( & 0 \\
\hline 18 & 4 & 84 & \\
\hline 0 & & ( & 0 \\
\hline & 0 & 0 & \\
\hline & & & \\
\hline
\end{tabular}

Using the techniques of [16], we find that the Hodge diamond of $G_{b o s} / J_{b o s}$ is

The proposal of [6] predicts that, for appropriately chosen fermionic weights $\left(q_{1}, q_{2}\right)=$ $\left(q_{1 *}, q_{2 *}\right), G_{b o s} / J_{b o s}$ corresponds to a Calabi-Yau supermanifold $M^{\left(q_{1}, q_{2}\right)}$ obtained as a crepant resolution of

$$
\left\{G^{\left(q_{1}, q_{2}\right)}=0\right\} \in \mathbf{W C P}_{\left(1,1,1,2,2,2,3 \mid q_{1}, q_{2}\right)[6]}^{6 \mid 2}
$$

where

$$
G^{\left(q_{1}, q_{2}\right)}=G_{b o s}+\xi_{1} \xi_{2} .
$$

The positive integer values of $\left(q_{1}, q_{2}\right)$ consistent with the quasihomogenity constraint

$$
q_{1}+q_{2}=6
$$

are (up to a relabelling of $\xi_{1}$ and $\xi_{2}$ )

$$
\left(q_{1}, q_{2}\right) \in A=\{(1,5),(2,4),(3,3)\} .
$$

The solutions for $\left(q_{1 *}, q_{2 *}\right)$ are those values of $\left(q_{1}, q_{2}\right) \in A$ for which the Hodge diamond of $M^{\left(q_{1}, q_{2}\right)}$ agrees with the Hodge diamond of $G_{b o s} / J_{b o s}$. It was found in [7] that the Hodge numbers obtained for $M^{\left(q_{1}, q_{2}\right)}$ by using the heuristic arguments of [6] do not agree with the Hodge numbers of $G_{b o s} / J_{b o s}$ for any $\left(q_{1}, q_{2}\right) \in A$. We can use the recently developed techniques of [13] to properly compute the Hodge diamond of $M^{\left(q_{1}, q_{2}\right)}$ for each $\left(q_{1}, q_{2}\right) \in A$.

According to (4.11), given $M^{\left(q_{1 *}, q_{2 *}\right)}$, for appropriately chosen fermionic weights

$$
\left(n_{\hat{\eta}_{1}}, n_{\hat{\gamma}_{1}}, n_{\hat{\eta}_{2}}, n_{\hat{\gamma}_{2}}\right)=\left(n_{\hat{\eta}_{1} *}, n_{\hat{\gamma}_{1} *}, n_{\hat{\eta}_{2} *}, n_{\hat{\gamma}_{2} *}\right)
$$

a Calabi-Yau supermanifold $\widetilde{M}_{\left(q_{1 *}, q_{2 *}\right)}^{\left(n_{\hat{\eta}_{1},}, n_{\hat{\gamma}_{1}}, n_{\hat{\eta}_{2}}, n_{\hat{\gamma}_{2}}\right)}$ obtained as a crepant resolution of

$$
\frac{\left\{\widetilde{W}^{\left(q_{1 *}, q_{2 *}\right)}=0\right\}}{\Gamma^{\left(q_{1 *}, q_{2 *}\right)} / \widetilde{J}^{\left(q_{1 *}, q_{2 *}\right)}} \subset \mathbf{W C P}_{\left(1,1,1,2,2,2,3, n_{x_{1}}^{\left(q_{1 *}, q_{2 *}\right)}, n_{x_{2}}^{\left(q_{1 *}, q_{2 *}\right)} \mid n_{\left.\hat{\eta}_{1}, n_{\hat{\gamma}_{1}}, n_{\hat{\eta}_{2}}, n_{\hat{\gamma}_{2}}\right)}\right) \mid 4}
$$


is mirror to $M^{\left(q_{1 *}, q_{2 *}\right)}$. From (4.10), we have that

$$
\widetilde{W}^{\left(q_{1 *}, q_{2 *}\right)}=\sum_{i=1}^{7} \prod_{j=1}^{7} y_{j}^{M_{j i}}+e^{t / 6} \prod_{j=1}^{7} y_{j} \prod_{b=1}^{2} x_{b}^{-1}+\sum_{a=1}^{2}\left(1+x_{a}^{-2} \hat{\eta}_{a} \hat{\gamma}_{a}\right) \prod_{b=1}^{2} x_{b}^{N_{b a}^{\left(q_{1 *}, q_{2 *}\right)}},
$$

where the matrix elements of the invertible matrices $\left(M_{j i}\right)$ and $\left(N_{b a}\right)^{\left(q_{1 *}, q_{2 *}\right)}$ of nonnegative integers are respectively given by

$$
\left(M_{j i}\right)=\operatorname{diag}(6,6,6,3,3,3,2)_{j i}, \quad 6=\sum_{a=1}^{2} N_{b a}^{\left(q_{1 *}, q_{2 *}\right)} q_{a *}
$$

as required by (4.6). Let $B^{\left(q_{1}, q_{2}\right)}$ be the set of values of $\left(n_{\hat{\eta}_{1}}, n_{\hat{\gamma}_{1}}, n_{\hat{\eta}_{2}}, n_{\hat{\gamma}_{2}}\right)$ for which $\widetilde{W}^{\left(q_{1}, q_{2}\right)}$ is quasihomogeneous of degree 6 . The solutions for $\left(n_{\hat{\eta}_{1} *}, n_{\hat{\gamma}_{1 *}}, n_{\hat{\eta}_{2} *}, n_{\hat{\gamma}_{2 *}}\right)$ are those values of $\left(n_{\hat{\eta}_{1}}, n_{\hat{\gamma}_{1}}, n_{\hat{\eta}_{2}}, n_{\hat{\gamma}_{2}}\right) \in B^{\left(q_{1 *}, q_{2 *}\right)}$ for which the Hodge diamond of $\widetilde{M}_{\left(q_{1 *}, q_{2 *}\right)}^{\left(n_{\hat{\eta}_{1}}, n_{\hat{\gamma}_{1}}, n_{\hat{\eta}_{2}}, n_{\hat{\gamma}_{2}}\right)}$ is what is expected for a mirror of $M^{\left(q_{1 *}, q_{2 *}\right)}$, i.e.

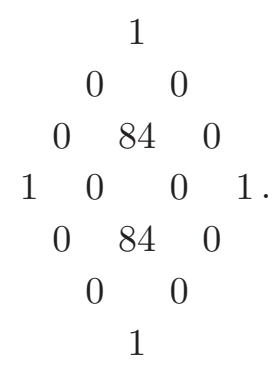

This is also what is expected for the Hodge diamond of the super Landau-Ginzburg orbifold $\widetilde{W}^{\left(q_{1 *}, q_{2 *}\right)} / \Gamma^{\left(q_{1 *}, q_{2 *}\right)}$. We can use the recently developed techniques of [13] to compute the Hodge diamond of $\widetilde{M}_{\left(q_{1 *}, q_{2 *}\right)}^{\left(n_{\hat{\gamma}_{1}}, n_{\hat{\gamma}_{1}}, n_{\hat{\eta}_{2}}, n_{\hat{\gamma}_{2}}\right)}$ for each $\left(n_{\hat{\eta}_{1}}, n_{\hat{\gamma}_{1}}, n_{\hat{\eta}_{2}}, n_{\hat{\gamma}_{2}}\right) \in B^{\left(q_{1 *}, q_{2 *}\right)}$.

Example 4.2. Consider the Landau-Ginzburg orbifold $G_{b o s} / J_{b o s}$, where

$$
G_{b o s}=\phi_{1}^{6}+\phi_{2}^{6}+\phi_{3}^{3}+\phi_{4}^{3}+\phi_{5}^{3}+\phi_{6}^{3}+\phi_{7}^{3}
$$

and $J_{b o s}$ is the diagonal subgroup of the phase symmetries of $G_{b o s}$, i.e.

$$
J_{\text {bos }}: \phi_{i} \rightarrow e^{2 \pi i Q_{i} / 6} \phi_{i} .
$$

Using the techniques of [16], we find that the Hodge diamond of $G_{b o s} / J_{b o s}$ is

$$
\begin{aligned}
& 1 \\
& 00 \\
& \begin{array}{lll}
0 & 1 & 0
\end{array} \\
& \begin{array}{llll}
1 & 73 & 73 & 1 .
\end{array} \\
& \begin{array}{lll}
0 & 1 & 0
\end{array} \\
& 00
\end{aligned}
$$


The proposal of [6] predicts that, for appropriately chosen fermionic weights $\left(q_{1}, q_{2}\right)=$ $\left(q_{1 *}, q_{2 *}\right), G_{b o s} / J_{b o s}$ corresponds to a Calabi-Yau supermanifold $M^{\left(q_{1}, q_{2}\right)}$ obtained as a crepant resolution of

$$
\left\{G^{\left(q_{1}, q_{2}\right)}=0\right\} \in \mathbf{W C P}_{\left(1,1,2,2,2,2,2 \mid q_{1}, q_{2}\right)[6]}^{6 \mid 2}
$$

where

$$
G^{\left(q_{1}, q_{2}\right)}=G_{b o s}+\xi_{1} \xi_{2} .
$$

The positive integer values of $\left(q_{1}, q_{2}\right)$ consistent with the quasihomogeneity constraint

$$
q_{1}+q_{2}=6
$$

are (up to a relabelling of $\xi_{1}$ and $\xi_{2}$ )

$$
\left(q_{1}, q_{2}\right) \in A=\{(1,5),(2,4),(3,3)\} .
$$

The solutions for $\left(q_{1 *}, q_{2 *}\right)$ are those values of $\left(q_{1}, q_{2}\right) \in A$ for which the Hodge diamond of $M^{\left(q_{1}, q_{2}\right)}$ agrees with the Hodge diamond of $G_{\text {bos }} / J_{\text {bos }}$. It was found in [6, 7] that the Hodge numbers obtained for $M^{\left(q_{1}, q_{2}\right)}$ by using the heuristic arguments of [6] agree with the Hodge numbers of $G_{b o s} / J_{b o s}$ when $\left(q_{1}, q_{2}\right)=(2,4)$ but disagree when $\left(q_{1}, q_{2}\right) \in\{(2,4),(3,3)\}$. We can use the recently developed techniques of [13] to properly compute the Hodge diamond of $M^{\left(q_{1}, q_{2}\right)}$ for each $\left(q_{1}, q_{2}\right) \in A$.

According to (4.11), given $M^{\left(q_{1 *}, q_{2 *}\right)}$, for appropriately chosen fermionic weights

$$
\left(n_{\hat{\eta}_{1}}, n_{\hat{\gamma}_{1}}, n_{\hat{\eta}_{2}}, n_{\hat{\gamma}_{2}}\right)=\left(n_{\hat{\eta}_{1 *}}, n_{\hat{\gamma}_{1} *}, n_{\hat{\eta}_{2 *}}, n_{\hat{\gamma}_{2} *}\right)
$$

a Calabi-Yau supermanifold $\widetilde{M}_{\left(q_{1 *}, q_{2 *}\right)}^{\left(n_{\hat{\eta}_{1},}, n_{\hat{\gamma}_{1}}, n_{\hat{\eta}_{2}}, n_{\hat{\gamma}_{2}}\right)}$ obtained as a crepant resolution of

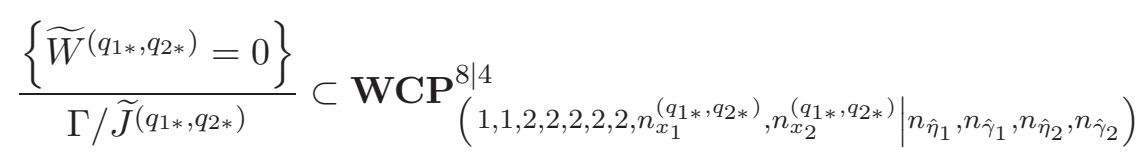

is mirror to $M^{\left(q_{1 *}, q_{2 *}\right)}$. From (4.10), we have that

$$
\widetilde{W}^{\left(q_{1 *}, q_{2 *}\right)}=\sum_{i=1}^{7} \prod_{j=1}^{7} y_{j}^{M_{j i}}+e^{t / 6} \prod_{j=1}^{7} y_{j} \prod_{b=1}^{2} x_{b}^{-1}+\sum_{a=1}^{2}\left(1+x_{a}^{-2} \hat{\eta}_{a} \hat{\gamma}_{a}\right) \prod_{b=1}^{2} x_{b}^{N_{b a}^{\left(q_{1 *}, q_{2 *}\right)}}
$$

where the matrix elements of the invertible matrices $\left(M_{j i}\right)$ and $\left(N_{b a}\right)^{\left(q_{1 *}, q_{2 *}\right)}$ of nonnegative integers are respectively given by

$$
\left(M_{j i}\right)=\operatorname{diag}(6,6,3,3,3,3,3)_{j i}, \quad 6=\sum_{a=1}^{2} N_{b a}^{\left(q_{1 *}, q_{2 *}\right)} q_{a *}
$$

as required by (4.6). Let $B^{\left(q_{1}, q_{2}\right)}$ be the set of values of $\left(n_{\hat{\eta}_{1}}, n_{\hat{\gamma}_{1}}, n_{\hat{\eta}_{2}}, n_{\hat{\gamma}_{2}}\right)$ for which $\widetilde{W}^{\left(q_{1}, q_{2}\right)}$ is quasihomogeneous of degree 6 . The solutions for $\left(n_{\hat{\eta}_{1} *}, n_{\hat{\gamma}_{1 *}}, n_{\hat{\eta}_{2 *} *}, n_{\hat{\gamma}_{2 *}}\right)$ are those values 
of $\left(n_{\hat{\eta}_{1}}, n_{\hat{\gamma}_{1}}, n_{\hat{\eta}_{2}}, n_{\hat{\gamma}_{2}}\right) \in B^{\left(q_{1 *}, q_{2 *}\right)}$ for which the Hodge diamond of $\widetilde{M}_{\left(q_{1 *}, q_{2 *}\right)}^{\left(n_{\hat{\eta}_{1}}, n_{\hat{\gamma}_{1}}, n_{\hat{\eta}_{2}}, n_{\hat{\gamma}_{2}}\right)}$ is what is expected for a mirror of $M^{\left(q_{1 *}, q_{2 *}\right)}$, i.e.

\begin{tabular}{|c|c|c|}
\hline \multicolumn{3}{|c|}{1} \\
\hline ( & & ) \\
\hline 0 & 73 & 0 \\
\hline 11 & & 1 \\
\hline 0 & 73 & 0 \\
\hline & & ) \\
\hline & 1 & \\
\hline
\end{tabular}

This is also what is expected for the Hodge diamond of the super Landau-Ginzburg orbifold $\widetilde{W}^{\left(q_{1 *}, q_{2 *}\right)} / \Gamma^{\left(q_{1 *}, q_{2 *}\right)}$. We can use the recently developed techniques of [13] to compute the Hodge diamond of $\widetilde{M}_{\left(q_{1 *}, q_{2 *}\right)}^{\left(n_{\hat{\eta}_{1}}, n_{\hat{\gamma}_{1}}, n_{\hat{\eta}_{2}}, n_{\hat{\gamma}_{2}}\right)}$ for each $\left(n_{\hat{\eta}_{1}}, n_{\hat{\gamma}_{1}}, n_{\hat{\eta}_{2}}, n_{\hat{\gamma}_{2}}\right) \in B^{\left(q_{1 *}, q_{2 *}\right)}$.

\section{Complete intersection in $\mathrm{WCP}^{m-1 \mid n}$}

Consider a member of the family of compact supervarieties $\mathbf{W C P}_{\left(Q_{1}, \ldots, Q_{m} \mid q_{1}, \ldots, q_{n}\right)\left[s_{1}, \ldots, s_{l}\right]}^{m-1 \mid n}$, i.e. a complete intersection of hypersurfaces, defined by the common zero locus $\cap_{\beta=1}^{l} G_{\beta}=0$ of quasihomogeneous polynomials $G_{\beta}=G_{\beta}(\phi, \xi)$ of degree $s_{\beta}$, in the weighted complex superprojective space of Section 2. A Kähler supermanifold may be obtained as a crepant resolution of this supervariety if a crepant resolution exists. Such a supermanifold would have nonnegative super-first Chern class when

$$
\sum_{i=1}^{m} Q_{i}-\sum_{a=1}^{n} q_{a}-\sum_{\beta=1}^{l} s_{\beta} \geq 0
$$

We will assume that a crepant resolution exists and the condition (5.1) is satisfied. To describe our Kähler supermanifold in terms of a gauged linear sigma model, we make the replacements

$$
\bar{P} e^{-2 s V} P \rightarrow \sum_{\beta=1}^{l} \bar{P}_{\beta} e^{-2 s_{\beta} V} P_{\beta}, \quad P \cdot G(\Phi, \Xi) \rightarrow \sum_{\beta=1}^{l} P_{\beta} \cdot G_{\beta}(\Phi, \Xi)
$$

in (4.2), where the chiral superfield $P_{\beta}$ has $U(1)$ charge $-s_{\beta}$ and $G_{\beta}(\Phi, \Xi)$ is a quasihomogeneous polynomial of $U(1)$ charge $s_{\beta}$. The resulting Lagrangian is

$$
\begin{aligned}
L_{\mathbf{W C P}_{\left(Q_{1}, \ldots, Q_{m} \mid q_{1}, \ldots, q_{n}\right)\left[s_{1}, \ldots, s_{l}\right]}^{m-1 \mid n}} & \int d^{4} \theta\left(\sum_{i=1}^{m} \bar{\Phi}_{i} e^{2 Q_{i} V} \Phi_{i}+\sum_{\beta=1}^{l} \bar{P}_{\beta} e^{-2 s_{\beta} V} P_{\beta}+\sum_{a=1}^{n} \bar{\Xi}_{a} e^{2 q_{a} V} \Xi_{a}-\frac{1}{2 e^{2}} \bar{\Sigma} \Sigma\right) \\
& -\frac{1}{2}\left(t \int d^{2} \tilde{\theta} \Sigma+\text { c.c. }\right)+\left(\int d^{2} \theta \sum_{\beta=1}^{l} P_{\beta} \cdot G_{\beta}(\Phi, \Xi)+\text { c.c. }\right) .
\end{aligned}
$$


The nonlinear sigma model phase is realized in the low energy limit with $r \gg 0, \sigma=0$, $p_{\beta}=0$, and target space

$$
\bigcap_{\beta=1}^{l}\left\{G_{\beta}=0\right\} \subset \frac{\left\{\left.\left(\phi_{1}, \ldots, \phi_{m} \mid \xi_{1}, \ldots, \xi_{n}\right)\left|\sum_{i=1}^{m} Q_{i}\right| \phi_{i}\right|^{2}+\sum_{a=1}^{n} q_{a}\left|\xi_{a}\right|^{2}=r\right\}}{U(1)}
$$

where $p_{\beta}$ is the lowest component of $P_{\beta}$. If the super Calabi-Yau condition

$$
\sum_{i=1}^{m} Q_{i}-\sum_{a=1}^{n} q_{a}=\sum_{\beta=1}^{l} s_{\beta}
$$

is satisfied, then the Fayet-Iliopoulos parameter $r$ does not renormalize.

Extending the result (4.5) to the present case, we obtain for the super Landau-Ginzburg mirror period

$$
\begin{aligned}
\Pi_{\widetilde{\mathbf{W C P}}_{\left(Q_{1}, \ldots, Q_{m} \mid q_{1}, \ldots, q_{n}\right)\left[s_{1}, \ldots s_{l}\right]}^{m-1 \mid n}} & \left(\prod_{i=1}^{m} d Y_{i}\right)\left(\prod_{\beta=1}^{l} d Y_{P_{\beta}} e^{-Y_{P_{\beta}}}\right)\left(\prod_{a=1}^{n} d X_{a} d \eta_{a} d \gamma_{a}\right) \\
= & \delta\left(\sum_{i=1}^{m} Q_{i} Y_{i}-\sum_{\beta=1}^{l} s_{\beta} Y_{P_{\beta}}-\sum_{a=1}^{n} q_{a} X_{a}-t\right) \\
& \times \exp \left[-\sum_{i=1}^{m} e^{-Y_{i}}-\sum_{\beta=1}^{l} e^{-Y_{P_{\beta}}}-\sum_{a=1}^{n} e^{-X_{a}}\left(1+\eta_{a} \gamma_{a}\right)\right],
\end{aligned}
$$

where $Y_{P_{\beta}}$ is a bosonic twisted chiral superfield (with periodicity $2 \pi i$ ) which satisfies

$$
\operatorname{Re} Y_{P_{\beta}}=\bar{P}_{\beta} e^{-2 s_{\beta} V} P_{\beta}
$$

\section{Complete intersection in a general toric supervariety}

Consider a member of the family of compact supervarieties $\mathcal{T}_{\left(Q_{A i} \mid q_{A a}\right)\left[s_{A \beta}\right]}^{m-k \mid n}$, i.e. a complete intersection of hypersurfaces, defined by the common zero locus $\cap_{\beta=1}^{l}\left\{G_{\beta}=0\right\}$ of quasihomogeneous polynomials $G_{\beta}=G_{\beta}(\phi, \xi)$ of multidegree $s_{A \beta}$, where $A=1, \ldots, k$, in the toric supervariety

$$
\mathcal{T}_{\left(Q_{A i} \mid q_{A a}\right)}^{m-k \mid n}=\frac{\mathbf{C}^{m \mid n} \backslash S}{\left(\mathbf{C}^{*}\right)^{k}}
$$

Here, the matrix elements of the matrices

$$
\left(Q_{A i} \mid q_{A a}\right)=\left(\begin{array}{ccc|ccc}
Q_{11} & \cdots & Q_{1 m} & q_{11} & \cdots & q_{1 n} \\
\vdots & & \vdots & \vdots & & \vdots \\
Q_{k 1} & \cdots & Q_{k m} & q_{k 1} & \cdots & q_{k n}
\end{array}\right), \quad\left[s_{A \beta}\right]=\left(\begin{array}{ccc}
s_{11} & \cdots & s_{1 l} \\
\vdots & & \vdots \\
s_{k 1} & \cdots & s_{k l}
\end{array}\right)
$$


are positive integers, $S$ is an exceptional supervariety in $\mathbf{C}^{m \mid n}$, and

$$
\begin{array}{ll}
\left(\mathbf{C}^{*}\right)^{k}: \phi_{i} \rightarrow \lambda^{Q_{A i}} \phi_{i}, \quad \xi_{a} \rightarrow \lambda^{q_{A a}} \xi_{a}, \quad i=1, \ldots, m ; & a=1, \ldots, n \\
A=1, \ldots, k ; \quad \lambda \in \mathbf{C}^{*}
\end{array}
$$

A Kähler supermanifold may be obtained as a crepant resolution of this supervariety if a crepant resolution exists. Such a supermanifold would have nonnegative super-first Chern class when

$$
\sum_{i=1}^{m} Q_{A i}-\sum_{a=1}^{n} q_{A a} \geq \sum_{\beta=1}^{l} s_{A \beta}, \quad A=1, \ldots, k
$$

We will assume that a crepant resolution exists and the condition (6.1) is satisfied. To describe our Kähler supermanifold in terms of of a gauged linear sigma model, we replace the $U(1)$ gauge group of (5.2) with $U(1)^{k}$. The resulting Lagrangian is

$$
\begin{aligned}
& L_{\mathcal{T}_{\left(Q_{A i} \mid q_{A a}\right)\left[s_{A \beta}\right]}^{m-k \mid n}} \\
& =\int d^{4} \theta\left(\sum_{i=1}^{m} \bar{\Phi}_{i} e^{2 \sum_{A=1}^{k} Q_{A i} V_{A}} \Phi_{i}+\sum_{\beta=1}^{l} \bar{P}_{\beta} e^{-2 \sum_{A=1}^{k} s_{A \beta} V_{A}} P_{\beta}\right. \\
& \left.\quad+\sum_{a=1}^{n} \bar{\Xi}_{a} e^{2 \sum_{A=1}^{k} q_{A a} V_{A}} \Xi_{a}-\sum_{A, B=1}^{k} \frac{1}{2 e_{A B}^{2}} \bar{\Sigma}_{A} \Sigma_{B}\right) \\
& -\frac{1}{2}\left(\int d^{2} \tilde{\theta} \sum_{A=1}^{k} t_{A} \Sigma_{A}+\text { c.c. }\right)+\left(\int d^{2} \theta \sum_{\beta=1}^{l} P_{\beta} \cdot G_{\beta}(\Phi, \Xi)+\text { c.c. }\right) .
\end{aligned}
$$

Under the $A$-th $U(1)$, the bosonic chiral superfields $\Phi_{i}$ have charges $Q_{A i}$, where $i=$ $1, \ldots, m$, the fermionic chiral superfields $\Xi_{a}$ have charges $q_{A a}$, where $a=1, \ldots, n$, the bosonic chiral superfields $P_{\beta}$ have charges $-s_{A \beta}$, and the quasihomogeneous polynomials $G_{\beta}=G_{\beta}(\Phi, \Xi)$ have charges $s_{A \beta}$, where $\beta=1, \ldots, l$. The nonlinear sigma model phase is realized in the low energy limit with $r_{A} \gg 0, \sigma_{A}=0, p_{\beta}=0$, and target space

$$
\bigcap_{\beta=1}^{l}\left\{G_{\beta}=0\right\} \subset \frac{\left\{\left.\left(\phi_{1}, \ldots, \phi_{m} \mid \xi_{1}, \ldots, \xi_{n}\right)\left|\sum_{i=1}^{m} Q_{A i}\right| \phi_{i}\right|^{2}+\sum_{a=1}^{n} q_{A a}\left|\xi_{a}\right|^{2}=r_{A}\right\}}{U(1)^{k}},
$$

where $\sigma_{A}$ is the lowest component of $\Sigma_{A}$. If the super Calabi-Yau condition

$$
\sum_{i=1}^{m} Q_{A i}-\sum_{a=1}^{n} q_{A a}=\sum_{\beta=1}^{l} s_{A \beta}, \quad A=1, \ldots, k
$$

is satisfied, then the $r_{A}$ do not renormalize. 
Extending the result (5.5) to the present case, we obtain the for super Landau-Ginzburg mirror period

$$
\begin{aligned}
\Pi_{\tilde{\mathcal{T}}_{\left.\left(Q_{A i} \mid q_{A a}\right) s_{A \beta}\right]}^{m-k \mid n}=} & \left(\prod_{i=1}^{m} d Y_{i}\right)\left(\prod_{\beta=1}^{l} d Y_{P_{\beta}} e^{-Y_{P_{\beta}}}\right)\left(\prod_{a=1}^{n} d X_{a} d \eta_{a} d \gamma_{a}\right) \\
& \times \prod_{A=1}^{k} \delta\left(\sum_{i=1}^{m} Q_{A i} Y_{i}-\sum_{\beta=1}^{l} s_{A \beta} Y_{P_{\beta}}-\sum_{a=1}^{n} q_{A a} X_{a}-t_{A}\right) \\
& \times \exp \left[-\sum_{i=1}^{m} e^{-Y_{i}}-\sum_{\beta=1}^{l} e^{-Y_{P_{\beta}}}-\sum_{a=1}^{n} e^{-X_{a}}\left(1+\eta_{a} \gamma_{a}\right)\right],
\end{aligned}
$$

where

$$
\begin{gathered}
\operatorname{Re} Y_{i}=\bar{\Phi}_{i} \exp \left(2 \sum_{A=1}^{k} Q_{A i} V_{A}\right) \Phi_{i}, \quad \operatorname{Re} Y_{P_{\beta}}=\bar{P}_{\beta} \exp \left(-2 \sum_{A=1}^{k} s_{A \beta} V_{A}\right) P_{\beta}, \\
\operatorname{Re} X_{a}=-\bar{\Xi}_{a} \exp \left(2 \sum_{A=1}^{k} q_{A a} V_{A}\right) \Xi_{a} .
\end{gathered}
$$

Consider making the change of variables

$$
e^{-Y_{P_{\beta}}}=\widetilde{P}_{\beta}, \quad e^{-Y_{i}}=U_{i} \prod_{\beta=1}^{l} \widetilde{P}_{\beta}^{M_{\beta i}}, \quad e^{-X_{a}}=V_{a} \prod_{\beta=1}^{l} \widetilde{P}_{\beta}^{N_{\beta a}},
$$

in (6.5), where the matrices $\left(M_{\beta i}\right)$ and $\left(N_{\beta a}\right)$ satisfy

$$
s_{A \beta}=\sum_{i=1}^{m} M_{\beta i} Q_{A i}-\sum_{a=1}^{n} N_{\beta a} q_{A a}, \quad A=1, \ldots, k ; \quad \beta=1, \ldots, l
$$

and, for fixed $i=\hat{\imath}, a=\bar{a}$, have at most one nonzero matrix element $M_{\hat{\beta} \hat{\imath}}=1, N_{\bar{\beta} \bar{a}}=1$, respectively. Then, in terms of the new variables, we obtain

$$
\begin{aligned}
& \Pi_{\widetilde{\mathcal{T}}_{\left(Q_{A i} \mid q_{A a}\right)\left[s_{A \beta}\right]}^{m-k \mid n}} \\
& =(-1)^{m+n+l} \int\left(\prod_{i=1}^{m} \frac{d U_{i}}{U_{i}}\right)\left(\prod_{\beta=1}^{l} d \widetilde{P}_{\beta}\right)\left(\prod_{a=1}^{n} \frac{d V_{a}}{V_{a}} d \eta_{a} d \gamma_{a}\right) \\
& \times \prod_{A=1}^{k} \delta\left(\ln \left(\frac{\prod_{i=1}^{m} U_{i}^{Q_{A i}}}{\prod_{a=1}^{n} V_{a}^{q_{A a}}}\right)+t_{A}\right) \\
& \times \exp \left[-\sum_{\beta=1}^{l} \widetilde{P}_{\beta}\left(\sum_{M_{\beta i}=1} U_{i}+\sum_{N_{\beta a}=1} V_{a}\left(1+\eta_{a} \gamma_{a}\right)+1\right)\right. \\
& \left.-\sum_{M_{\beta i}=0 \forall \beta} U_{i}-\sum_{N_{\beta a}=0 \forall \beta} V_{a}\left(1+\eta_{a} \gamma_{a}\right)\right] \text {. }
\end{aligned}
$$


Performing the integration over the $\widetilde{P}_{\beta}$ yields

$$
\begin{aligned}
\prod_{\tilde{\mathcal{T}}_{\left(Q_{A i}^{m} \mid q_{A a}\right)\left[s_{A \beta}\right]}^{m-k \mid n}} & \left(\prod_{i=1}^{m} \frac{d U_{i}}{U_{i}}\right)\left(\prod_{a=1}^{n} \frac{d V_{a}}{V_{a}} d \eta_{a} d \gamma_{a}\right) \prod_{A=1}^{k} \delta\left(\ln \left(\frac{\prod_{i=1}^{m} U_{i}^{Q_{A i}}}{\prod_{a=1}^{n} V_{a}^{q_{A a}}}\right)+t_{A}\right) \\
& \times \prod_{\beta=1}^{l} \delta\left(\sum_{M_{\beta i}=1} U_{i}+\sum_{N_{\beta a}=1} V_{a}\left(1+\eta_{a} \gamma_{a}\right)+1\right) \\
& \times \exp \left(-\sum_{M_{\beta i}=0 \forall \beta} U_{i}-\sum_{N_{\beta a}=0 \forall \beta} V_{a}\left(1+\eta_{a} \gamma_{a}\right)\right) .
\end{aligned}
$$

Thus, we have obtained an $(m-k-l-n)$-dimensional noncompact supermanifold $\widetilde{M}^{\circ} \subset$ $\left(\mathbf{C}^{*}\right)^{m+n \mid 2 n}$ defined by

$$
\prod_{i=1}^{m} U_{i}^{Q_{A i}} \prod_{a=1}^{n} V_{a}^{-q_{A a}}=e^{-t_{A}}, \quad \sum_{M_{\beta i}=1} U_{i}+\sum_{N_{\beta a}=1} V_{a}\left(1+\eta_{a} \gamma_{a}\right)+1=0 .
$$

The period (6.9) is identical to the period of a super Landau-Ginzburg model on $\widetilde{M}^{\circ}$ with superpotential

$$
\widetilde{W}_{\widetilde{M}^{\circ}}=\sum_{M_{\beta i}=0 \forall \beta} U_{i}+\sum_{N_{\beta a}=0 \forall \beta} V_{a}\left(1+\eta_{a} \gamma_{a}\right) .
$$

Example 6.1. Consider a gauged linear sigma model corresponding to the complete intersection defined by two quadrics in $\mathbf{C P}^{3 \mid 2}$. We must find matrices $\left(M_{\beta i}\right)$ and $\left(N_{\beta a}\right)$ which satisfy (6.8) and, for fixed $i=\hat{\imath}, a=\bar{a}$, have at most one nonzero matrix element $M_{\hat{\beta} \hat{\imath}}=1$, $N_{\bar{\beta} \bar{a}}=1$, respectively. We can choose

$$
\left(M_{\beta i}\right)=\left(\begin{array}{llll}
1 & 1 & 0 & 0 \\
0 & 0 & 1 & 1
\end{array}\right), \quad\left(N_{\beta a}\right)=\left(\begin{array}{ll}
0 & 0 \\
0 & 0
\end{array}\right) .
$$

The mirror theory is thus a super Landau-Ginzburg model on the supermanifold $\widetilde{M}^{\circ} \subset$ $\left(\mathbf{C}^{*}\right)^{(6 \mid 4)}$ defined by

$$
U_{1} U_{2} U_{3} U_{4} V_{1}^{-1} V_{2}^{-1}=e^{-t}, \quad U_{1}+U_{2}+1=0, \quad U_{3}+U_{4}+1=0
$$

and with superpotential

$$
\widetilde{W}_{\widetilde{M}^{\circ}}=V_{1}\left(1+\eta_{1} \gamma_{1}\right)+V_{2}\left(1+\eta_{2} \gamma_{2}\right) .
$$

\section{Period relations}

In this section, we will establish some relations between periods of mirrors of gauged linear sigma models corresponding to various geometries. These relations generalize results which were obtained in [14] by working with A-models. 
Starting from (4.5), we obtain

$$
\begin{aligned}
& \Pi_{\widetilde{\mathbf{W C P}}_{\left(Q_{1}, \ldots, Q_{m} \mid q_{1}, \ldots, q_{n}\right)[s]}^{m-1 \mid n}} \int\left(\prod_{i=1}^{m} d Y_{i}\right) d Y_{P} e^{-Y_{P}}\left(\prod_{a=1}^{n} d X_{a} d \eta_{a} d \gamma_{a}\right) \delta\left(\sum_{i=1}^{m} Q_{i} Y_{i}-s Y_{P}-\sum_{a=1}^{n} q_{a} X_{a}-t\right) \\
& \quad \times \exp \left[-\sum_{i=1}^{m} e^{-Y_{i}}-e^{-Y_{P}}-\sum_{a=1}^{n} e^{-X_{a}}\left(1+\eta_{a} \gamma_{a}\right)\right] \\
&=(-1)^{n} \int\left(\prod_{i=1}^{m} d Y_{i}\right) d Y_{P} e^{-Y_{P}}\left(\prod_{a=1}^{n} d X_{a} e^{-X_{a}}\right) \delta\left(\sum_{i=1}^{m} Q_{i} Y_{i}-s Y_{P}-\sum_{a=1}^{n} q_{a} X_{a}-t\right) \\
& \quad \times \exp \left(-\sum_{i=1}^{m} e^{-Y_{i}}-e^{-Y_{P}}-\sum_{a=1}^{n} e^{-X_{a}}\right) \\
&=\Pi_{\widetilde{\mathbf{W C P}}_{\left(Q_{1}, \ldots, Q_{m}\right)\left[s, q_{1}, \ldots, q_{n}\right]}^{m-1} .}
\end{aligned}
$$

Thus,

$$
\Pi_{\widetilde{\mathbf{W C P}_{\left(Q_{1}, \ldots, Q_{m} \mid q_{1}, \ldots, q_{n}\right)[s]}^{m-1 \mid n}}}^{m}=\Pi_{\widetilde{\mathbf{W C P}}_{\left(Q_{1}, \ldots, Q_{m}\right)\left[s, q_{1}, \ldots, q_{n}\right]}^{m-1}}^{m .}
$$

Similarly, starting from (5.5), we obtain

$$
\begin{aligned}
& \Pi_{\widetilde{\mathbf{W C P}}_{\left(Q_{1}, \ldots, Q_{m} \mid q_{1}, \ldots, q_{n}\right)\left[s_{1}, \ldots, s_{l}\right]}^{m-1 \mid n}} \\
& =\int\left(\prod_{i=1}^{m} d Y_{i}\right)\left(\prod_{\beta=1}^{l} d Y_{P_{\beta}} e^{-Y_{P_{\beta}}}\right)\left(\prod_{a=1}^{n} d X_{a} d \eta_{a} d \gamma_{a}\right) \\
& \times \delta\left(\sum_{i=1}^{m} Q_{i} Y_{i}-\sum_{\beta=1}^{l} s_{\beta} Y_{P_{\beta}}-\sum_{a=1}^{n} q_{a} X_{a}-t\right) \\
& \times \exp \left[-\sum_{i=1}^{m} e^{-Y_{i}}-\sum_{\beta=1}^{l} e^{-Y_{P_{\beta}}}-\sum_{a=1}^{n} e^{-X_{a}}\left(1+\eta_{a} \gamma_{a}\right)\right] \\
& =(-1)^{l-1} \int\left(\prod_{i=1}^{m} d Y_{i}\right) d Y_{P_{l}} e^{-Y_{P_{l}}}\left(\prod_{\beta=1}^{l-1} d Y_{P_{\beta}} \eta_{P_{\beta}} \gamma_{P_{\beta}}\right)\left(\prod_{a=1}^{n} d X_{a} d \eta_{a} d \gamma_{a}\right) \\
& \times \delta\left(\sum_{i=1}^{m} Q_{i} Y_{i}-s_{l} Y_{P_{l}}-\sum_{\beta=1}^{l-1} s_{\beta} Y_{P_{\beta}}-\sum_{a=1}^{n} q_{a} X_{a}-t\right) \\
& \times \exp \left[-\sum_{i=1}^{m} e^{-Y_{i}}-e^{-Y_{P_{l}}}-\sum_{\beta=1}^{l-1} e^{-Y_{P_{\beta}}}\left(1+\eta_{P_{\beta}} \gamma_{P_{\beta}}\right)-\sum_{a=1}^{n} e^{-X_{a}}\left(1+\eta_{a} \gamma_{a}\right)\right]
\end{aligned}
$$




$$
\begin{aligned}
& =\Pi_{\widetilde{\mathbf{W C P}}_{\left(Q_{1}, \ldots, Q_{m} \mid q_{1}, \ldots, q_{n}, s_{1}, \ldots, s_{l-1}\right)\left[s_{l}\right]}^{m-1 \mid n+l-1}} \\
& =(-1)^{l} \int\left(\prod_{i=1}^{m} d Y_{i}\right)\left(\prod_{\beta=1}^{l} d Y_{P_{\beta}} \eta_{P_{\beta}} \gamma_{P_{\beta}}\right)\left(\prod_{a=1}^{n} d X_{a} d \eta_{a} d \gamma_{a}\right) \\
& \quad \times \delta\left(\sum_{i=1}^{m} Q_{i} Y_{i}-\sum_{\beta=1}^{l} s_{\beta} Y_{P_{\beta}}-\sum_{a=1}^{n} q_{a} X_{a}-t\right) \\
& \quad \times \exp \left[-\sum_{i=1}^{m} e^{-Y_{i}}-\sum_{\beta=1}^{l} e^{\left.-Y_{P_{\beta}}\left(1+\eta_{P_{\beta}} \gamma_{P_{\beta}}\right)-\sum_{a=1}^{n} e^{-X_{a}}\left(1+\eta_{a} \gamma_{a}\right)\right]}\right. \\
& =\prod_{\widetilde{\mathbf{W C P}}_{\left(Q_{1}, \ldots, Q_{m} \mid q_{1}, \ldots, q_{n}, s_{1}, \ldots, s_{l}\right)}^{m-1 \mid n+l}} \cdot
\end{aligned}
$$

Thus,

$$
\begin{aligned}
& \Pi_{\widetilde{\operatorname{WCP}}_{\left(Q_{1}, \ldots, Q_{m} \mid q_{1}, \ldots, q_{n}\right)\left[s_{1}, \ldots, s_{l}\right]}^{m-1 \mid n}}=\Pi_{\left.\widetilde{\mathbf{W C P}}_{\left(Q_{1}, \ldots, Q_{m} \mid q_{1}, \ldots, q_{n}, s_{1}, \ldots, s_{l-1}\right)\left[s_{l}\right]}^{m-1 \mid n+l}\right]} \\
& =\Pi_{\widetilde{\mathbf{W C P}}_{\left(Q_{1}, \ldots, Q_{m} \mid q_{1}, \ldots, q_{n}, s_{1}, \ldots, s_{l}\right)}^{m-1 \mid n+l}}^{m .}
\end{aligned}
$$

In a straightforward manner, one can generalize the relation (7.1) to obtain

$$
\Pi_{\widetilde{\mathcal{T}}_{\left(Q_{A i} \mid q_{A a}\right)\left[s_{A}\right]}^{m-k \mid n}}=\Pi_{\widetilde{\mathcal{T}}_{\left(Q_{A i}\right)\left[s_{A}, q_{A a}\right]}^{m \mid n}}
$$

and generalize (7.2) to obtain

$$
\Pi_{\widetilde{\mathcal{T}}_{\left(Q_{A i} \mid q_{A a}\right)\left[s_{A 1}, \ldots, s_{A l}\right]}^{m \mid n}}=\Pi_{\widetilde{\mathcal{T}}_{\left(Q_{A i} \mid q_{A a}, s_{A 1}, \ldots, s_{A l-1}\right)\left[s_{A l}\right]}^{m \mid n}}=\Pi_{\widetilde{\mathcal{T}}_{\left(Q_{A i} \mid q_{A a},{ }^{s}{ }_{A 1}, \ldots, s_{A l}\right)}^{m-k \mid}} .
$$

\section{Categorical interpretation}

In this section, we give a categorical intepretation of the material presented in Sections $2-7$. We briefly review the phenomena of spectra and gaps [17] and connect them with supercohomology calculations and algebraic cycles. Complete details will appear in [13].

\subsection{Supermanifold cohomology calculations}

We begin with a simple example.

Example 8.1. Consider a hypersurface

$$
M=\left\{\phi_{1}^{3}+\phi_{2}^{3}+\phi_{3}^{3}+\phi_{4}^{3}+\phi_{5}^{3}+\xi_{1} \xi_{2}=0\right\} \in \mathbf{W C P}_{(1,1,1,1,1 \mid 1,2)[3]}^{4 \mid 2} .
$$

Treating $M$ as a DG scheme, its structure sheaf becomes the complex shown below. 


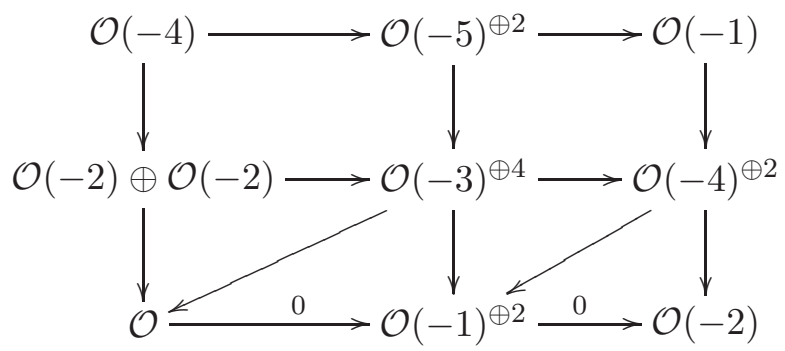

Note that

$$
h^{1}(\mathcal{O})=h^{1}\left(K_{\mathbf{C P}^{5}}-2 H-2 H-2 H\right)=1 .
$$

Here $H$ is the hyperplane section in $\mathbf{C P}^{5}$. Applying the Riemman-Roch theorem, we obtain

$$
h^{2,1}(M)=5, \quad h^{1,1}(M)=1 .
$$

Example 8.1 gives us a flavor of supermanifold cohomology calculations. In general, each supermanifold Hodge number is the sum of a number obtained from a DG scheme calculation and a number coming from the gaps of the category of singularities; see Table 1 and Definition 8.2. Further details can be found in [13].

On the symplectic side, the Example 8.1 calculation can be seen tropically as [18]:

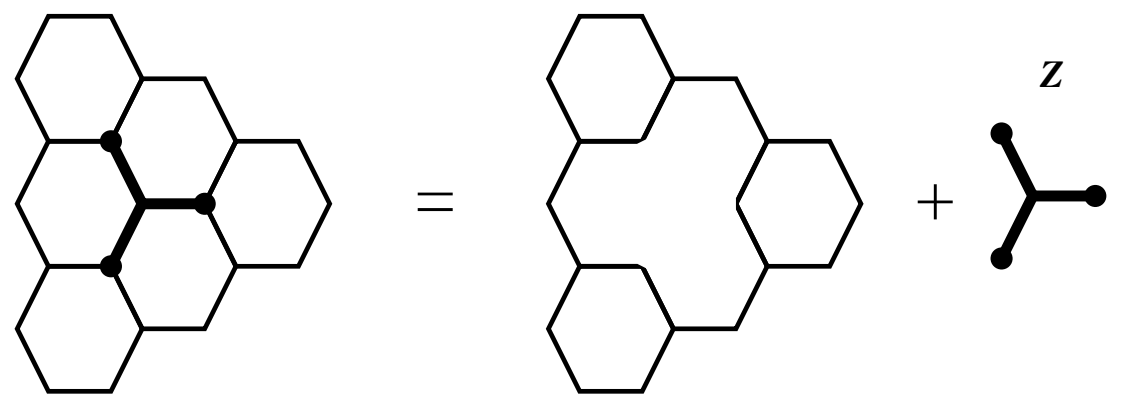

Here, $Z$ is the mirror of $\mathbf{W C P}^{1 \mid 2}$. We thus have the A-side categorical equivalence

$$
\operatorname{Fuk}(M)^{d}=\langle\operatorname{Fuk}(\text { genus } 4 \text { curve), } \operatorname{Fuk}(Z), \ldots\rangle,
$$

where Fuk denotes the Fukaya category. In other words, the B-side noncommutative deformation corresponds to a conifold transition, which produces the Fukaya category of a genus 4 curve and the mirror of $\mathbf{W C P}^{1 \mid 2}$. This is a special case of Theorem 8.2.

\subsection{Super and DG scheme version of Homological Mirror Symmetry}

In what follows, $D^{b}(A)$ is the bounded derived category of coherent sheaves on the scheme $A, D^{b}(A)^{d}$ is a (possibly trivial) deformation of $D^{b}(A)$, and $\left\langle D^{b}(A), \ldots\right\rangle$ is a semiorthogonal decomposition which includes $D^{b}(A)$ as a summand. Similarly, $\operatorname{Fuk}(A)^{d}$ is a (possibly trivial) deformation of $\operatorname{Fuk}(A)$ and $\langle\operatorname{Fuk}(A), \ldots\rangle$ is a semiorthogonal decomposition which includes $\operatorname{Fuk}(A)$ as a summand. 


\section{Theorem 8.1.}

$$
D^{b}\left(\mathbf{W C P}_{\left(Q_{1}, \ldots, Q_{m} \mid q_{1}, \ldots, q_{n}\right)}^{m-1 \mid n}\right)^{d}=\left\langle D^{b}\left(\mathbf{W C P}_{\left(Q_{1}, \ldots, Q_{m}\right)\left[q_{1}, \ldots, q_{n}\right]}^{m-1}\right), \ldots\right\rangle .
$$

The proof follows from [19] and is based on the identity

$$
\mathbf{C}\left[\phi_{1}, \ldots \phi_{m}, \xi_{1}, \ldots, \xi_{n}\right]_{((u))}=\mathbf{C}\left[\phi_{k+1}, \ldots, \phi_{m}\right]_{((u))} \otimes \prod_{j=1}^{k} \mathbf{C}\left[\phi_{j}, \xi_{1}, \ldots, \xi_{n}\right]
$$

Theorem 8.1 gives a new proof of a theorem due to Bardelli and Müller-Stach [20]. We can restate Theorem 8.1 by saying that some noncommutative deformation of $D^{b}\left(\mathbf{W C P}_{\left(Q_{1}, \ldots, Q_{m} \mid q_{1}, \ldots, q_{n}\right)}^{m-1 \mid n}\right)$ yields $D^{b}\left(\mathbf{W C P}_{\left(Q_{1}, \ldots, Q_{m}\right)\left[q_{1}, \ldots, q_{n}\right]}^{m-1}\right)$. Alternatively, we can say that $D^{b}\left(\mathbf{W C P}_{\left(Q_{1}, \ldots, Q_{m} \mid q_{1}, \ldots, q_{n}\right)}^{m-1 \mid n}\right)$ contains as a semiorthogonal summand a noncommutative Calabi-Yau (or Fano or general type manifold). The following theorem is the A-side version of this statement.

\section{Theorem 8.2.}

$$
\operatorname{Fuk}\left(\mathbf{W C P}_{\left(Q_{1}, \ldots, Q_{n} \mid q_{1}, \ldots, q_{n}\right)}^{m-1 \mid n}\right)^{d}=\left\langle\operatorname{Fuk}\left(\mathbf{W C P}_{\left(Q_{1}, \ldots, Q_{m}\right)\left[q_{1}, \ldots, q_{n}\right]}^{m-1}\right), \ldots\right\rangle .
$$

Figure 1 gives a schematic picture of classical Homological Mirror Symmetry in the version which is relevant for our purpose. For more details, see [18]. Theorems 8.1 and 8.2 can be seen as an extension of Homological Mirror Symmetry to the case of DG schemes. More details will appear in [13].

Remark 8.1. Theorem 8.2 can be seen as a symplectic version of Orlov's theorem [19].

Remark 8.2. Theorem 8.2 can be extended to hypersurfaces and complete intersections in general toric supervarieties.

Our categorical findings are summarized in Figures 2 and 3. These figures give a natural super generalization of classical Homological Mirror Symmetry.

Remark 8.3. Theorems 8.1 and 8.2 suggest that different Landau-Ginzburg models can be associated to different noncommutative Hodge substructures in the Hochshild homology of $D^{b}$ or Fukaya categories.

\subsection{Algebro-geometric applications}

The above extension of classical Homological Mirror Symmetry to the case of supermanifolds and DG schemes suggests some applications which we discuss next.

Definition 8.1 (Fano CY). We call a manifold a Fano CY manifold if its Hodge diamond contains the Hodge structure of a $C Y$ (possibly noncommutative) manifold; see Figure 4. 


\begin{tabular}{l}
\hline A-models (symplectic) \\
\hline$X=(X, \omega)$ a closed symplectic manifold \\
Fukaya category Fuk $(X)$ : Objects are \\
Lagrangian submanifolds $L$ which may \\
be equipped with flat line bundles. Mor- \\
phisms are given by Floer cohomology \\
$\operatorname{HF}^{*}\left(L_{0}, L_{1}\right)$.
\end{tabular}

B-models (algebraic)

$X$ a smooth projective variety

Derived category $D^{b}(X)$ : Objects are complexes of coherent sheaves $\mathcal{E}$. Morphisms are $\operatorname{Ext}^{*}\left(\mathcal{E}_{0}, \mathcal{E}_{1}\right)$.

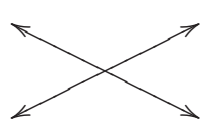

$Y$ a non-compact symplectic manifold with a proper map $W: Y \rightarrow \mathbf{C}$ which is a symplectic fibration with singularities.

Fukaya-Seidel category of the LandauGinzburg model FS(LG): Objects are Lagrangian submanifolds $L \subset Y$ which, at infinity, are fibered over $\mathbf{R}^{+} \subset \mathbf{C}$. The morphisms are $H F^{*}\left(L_{0}^{+}, L_{1}\right)$, where the superscript + indicates a perturbation removing intersection points at infinity.

$Y$ a smooth quasi-projective variety with a proper holomorphic map $W: Y \rightarrow \mathbf{C}$.

The category $D_{\text {sing }}^{b}(W)$ of algebraic $B$ branes which is obtained by considering the singular fibers $Y_{z}=W^{-1}(z)$, dividing $D^{b}\left(Y_{z}\right)$ by the subcategory of perfect complexes $\operatorname{Perf}\left(Y_{z}\right)$, and then taking the direct sum over all such $z$.

Figure 1. Classical Homological Mirror Symmetry

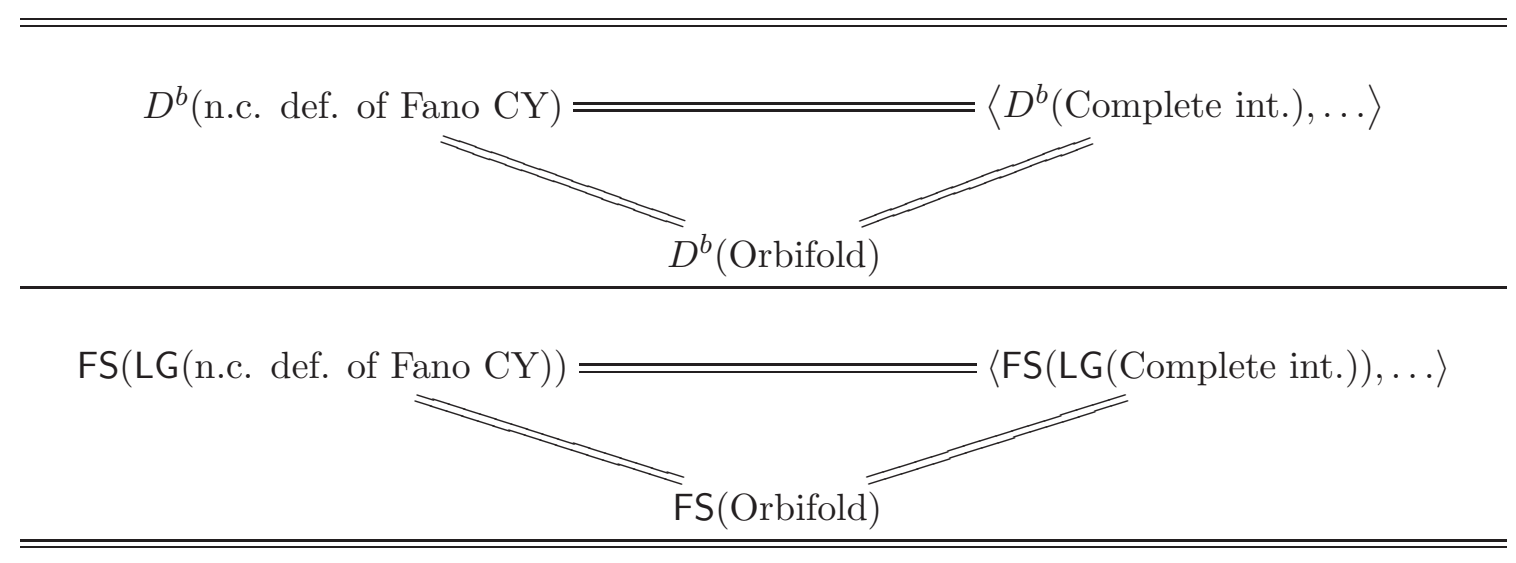

Figure 2. General picture of noncommutative deformations

Example 8.2. $X=\left\{\phi_{1}^{3}+\cdots+\phi_{9}^{3}=0\right\} \subset \mathbf{C P}^{8}$ is a seven-dimensional Fano $C Y$.

Theorem 8.3. Let $X$ be the Fano $C Y$ defined in Example 8.2. Then $G_{3}(X)$ is infinitely generated. 


\begin{tabular}{c|c}
\hline \hline$D^{b}\left(\mathbf{W C P}_{\left(Q_{1}, \ldots, Q_{m} \mid q_{1}, \ldots, q_{n}\right)[s]}^{m-1 \mid n}\right)^{d}$ & $\left\langle D^{b}\left(\mathbf{W C P}_{\left(Q_{1}, \ldots, Q_{m}\right)\left[s, q_{1}, \ldots, q_{n}\right]}^{m-1}\right), \ldots\right\rangle$ \\
\hline $\mathrm{FS}\left(\mathrm{LG}\left(\mathbf{W C P}_{\left(Q_{1}, \ldots, Q_{m} \mid q_{1}, \ldots, q_{n}\right)[s]}^{m-1 \mid n}\right)\right)^{d}$ & $\left\langle\mathrm{FS}\left(\operatorname{LG}\left(\mathbf{W C P}_{\left(Q_{1}, \ldots, Q_{m}\right)\left[s, q_{1}, \ldots, q_{n}\right]}^{m-1}\right)\right), \ldots\right\rangle$ \\
\hline \hline
\end{tabular}

Figure 3. Super Homological Mirror Symmetry

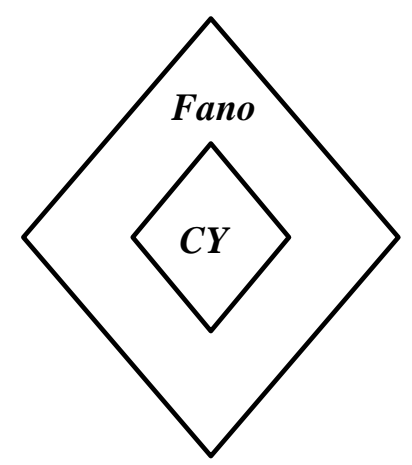

Figure 4. Definition of Fano CY manifold

Proof. It follows from Theorem 8.1 that

$$
D^{b}\left(X+\xi_{1} \xi_{2}+\xi_{3} \xi_{4}\right)=D^{b}(N C Y)
$$

Here, $N C Y$ is a noncommutative Calabi-Yau of superdimension three with a DG scheme structure coming from the anticommuting variables $\xi_{1}, \xi_{2}, \xi_{3}, \xi_{4}$. Thus,

$$
G r_{3}(X)=G r_{1}(N C Y) \text {. }
$$

It follows from [22] that we have a super Calabi-Yau integrable system arising from the universal variation of Hodge structures of the noncommutative Calabi-Yau manifold. A noncommutative modification of a theorem of Voisin [21] applied to the above integrable system yields the result that $G r_{1}(N C Y)$ is infinitely generated.

The above argument applies to many other examples. For more details see [22].

\subsection{Gaps and spectra}

In this subsection, we review the notions of spectra and gaps following [17].

Noncommutative Hodge structures were introduced in [23] as a means of bringing the techniques and tools of Hodge theory into the categorical and noncommutative realm. In the classical setting, much of the information about an isolated singularity is contained in the Hodge spectrum (a set of rational eigenvalues of the monodromy operator). A categorical analogue of the Hodge spectrum appears in the works of Orlov [24] and Rouqier [25]. Let us call this analogue the Orlov spectrum (a rigorous definition will appear below). 
Very little is known about the Orlov spectrum. However, recent work [17] suggests an intimate connection with the classical theory.

Let us recall the definition of the Orlov spectrum and discuss some of the main results in [17]. Let $\mathcal{T}$ be a triangulated category. For any $G \in \mathcal{T}$, denote by $\langle G\rangle_{0}$ the smallest full subcategory containing $G$ which is closed under isomorphisms, shifting, and taking finite direct sums and summands. Now, inductively define $\langle G\rangle_{n}$ as the full subcategory of objects $B$ such that there is a distinguished triangle $X \rightarrow B \rightarrow Y \rightarrow X$, with $X \in\langle G\rangle_{n-1}$ and $Y \in\langle G\rangle_{0}$.

Definition 8.2. Let $G$ be an object of a triangulated category $\mathcal{T}$. If there is an $n$ with $\langle G\rangle_{n}=\mathcal{T}$, we set

$$
\Theta(G):=\min \left\{n \geq 0 \mid\langle G\rangle_{n}=\mathcal{T}\right\} .
$$

Otherwise, we set $\Theta(G):=\infty$. We call $\Theta(G)$ the generation time of $G$. If $\Theta(G)$ is finite, we say that $G$ is a strong generator. The Orlov spectrum of $\mathcal{T}$ is the union of all possible generation times for strong generators of $\mathcal{T}$. The Rouquier dimension is the smallest number in the Orlov spectrum. We say that a triangulated category $\mathcal{T}$ has a gap of length $s$, if $a$ and $a+s+1$ are in the Orlov spectrum but $r$ is not in the Orlov spectrum for $a<r<a+s+1$.

Conjecture 8.1. If $X$ is a smooth variety then any gap of $D^{b}(X)$ has length at most the Krull dimension of $X$.

In the noncommutative situation, the gaps could be larger. We will now explain how the notions of gaps and spectra connect with supercohomology calculations.

\subsection{Supermanifolds and exotic $(p, p)$ cycles}

In this section, we look at Example 4.1 and Example 4.2 from the perspective of the Hodge conjecture, i.e. every $(p, p)$ cycle is algebraic. We begin with the following.

Conjecture 8.2. ("super matrix factorization" version of the Hodge conjecture; see [26]) Every $(p, p)$ class in the Jacobian ideal (Hochschild cohomology) acts nontrivially on the category of singularities.

Conjecture 8.3. There is a correspondence between "missing" supermanifold Hodge numbers and increasing gaps of categories of singularities.

The supermanifolds of Examples 4.1 and 4.2 are candidates for displaying the behavior described in Conjecture 8.3. Other candidates may be obtained by following the procedure described in $[6,7]$.

Conjectures 8.2 and 8.3 are based on the (not yet completely established) equality

$$
\Theta(G)=\Theta\left(G+G^{\prime}\right)+B\left(G^{\prime}\right) \cdot l_{G^{\prime}}(G)-M E .
$$

Here, $l_{G^{\prime}}(G)$ is the number of steps in which $G^{\prime}$ generates $G, B\left(G^{\prime}\right)$ measures how far $\operatorname{End}\left(G^{\prime}\right)$ is from being formal, and $M E$ is the monodromy effect on the mirror side. 


\begin{tabular}{|c|c|}
\hline $\begin{array}{c}\text { Supermanifolds } \\
M=\left\{\phi_{1}^{3}+\cdots+\phi_{9}^{3}+\xi_{1} \xi_{2}+\xi_{3} \xi_{4}=0\right\} \\
\in \mathbf{W C P}_{\left(1,1,1,1,1,1,1,1,1 \mid q_{1}, q_{2}, q_{3}, q_{4}\right)[3]}^{8 \mid 4}\end{array}$ & $\begin{array}{c}X=\left\{\phi_{1}^{3}+\cdots+\phi_{9}^{3}=0\right\} \subset \mathbf{C P}^{8} \\
\operatorname{Gap}\left(D^{b}(X)\right)=6 \\
\operatorname{Gap}\left(D^{b}(M)\right)=4\end{array}$ \\
\hline 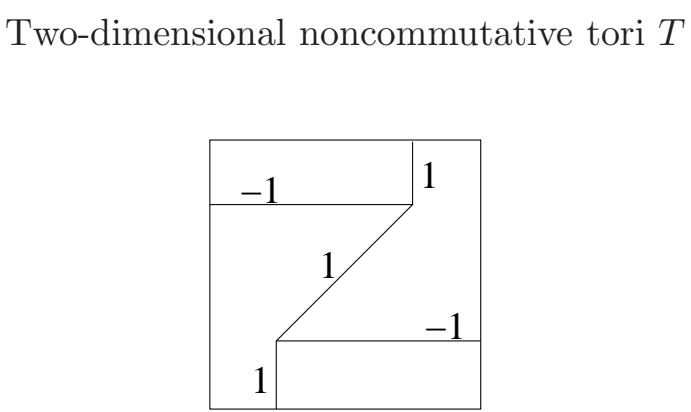 & $\begin{array}{c}\operatorname{Gap}(\operatorname{Fuk}(\text { Generic }))=\infty \\
\operatorname{Gap}(\operatorname{Fuk}(T))=3\end{array}$ \\
\hline Too few Lagrangian spheres in & $\begin{array}{c} \\
\text { Gap }(\text { Generic })=n \\
\text { Gap }(\text { Special })=n-k, k<2\end{array}$ \\
\hline
\end{tabular}

Table 1. Gap drops

Remark 8.4. Theorem 8.1 and Equation (8.1) explains the connection with the supercohomology calculations indicated in Examples 4.1 and 4.2. Indeed what needs to be computed there are the Hochshild cohomologies of deformed categories; gaps are responsible for "missing" Hodge numbers. In order to do the calculations, one needs to introduce an enhanced notion of spectra. This will be explained in detail in [13].

Based on Conjectures 8.2 and 8.3, two kinds of spectral anomalies are possible:

1. Big gap drops as a consequence of appearance of new $(p, p)$ classes. The first two rows of Table 1 and the tropical Abelian varieties of [18] are examples.

2. Preservation of big gaps, i.e. rather small disappearance of gaps as a consequence of new $(p, p)$ classes. The third row of Table 1 is an example.

In Table 2, we connect small gap drops with coniveau filtration; see [27]. The first row of the table explains the action of elements of the Jacobian ring and tropical $(p, p)$ cycles on the category of matrix factorizations and Fukaya categories, respectively. The trace 


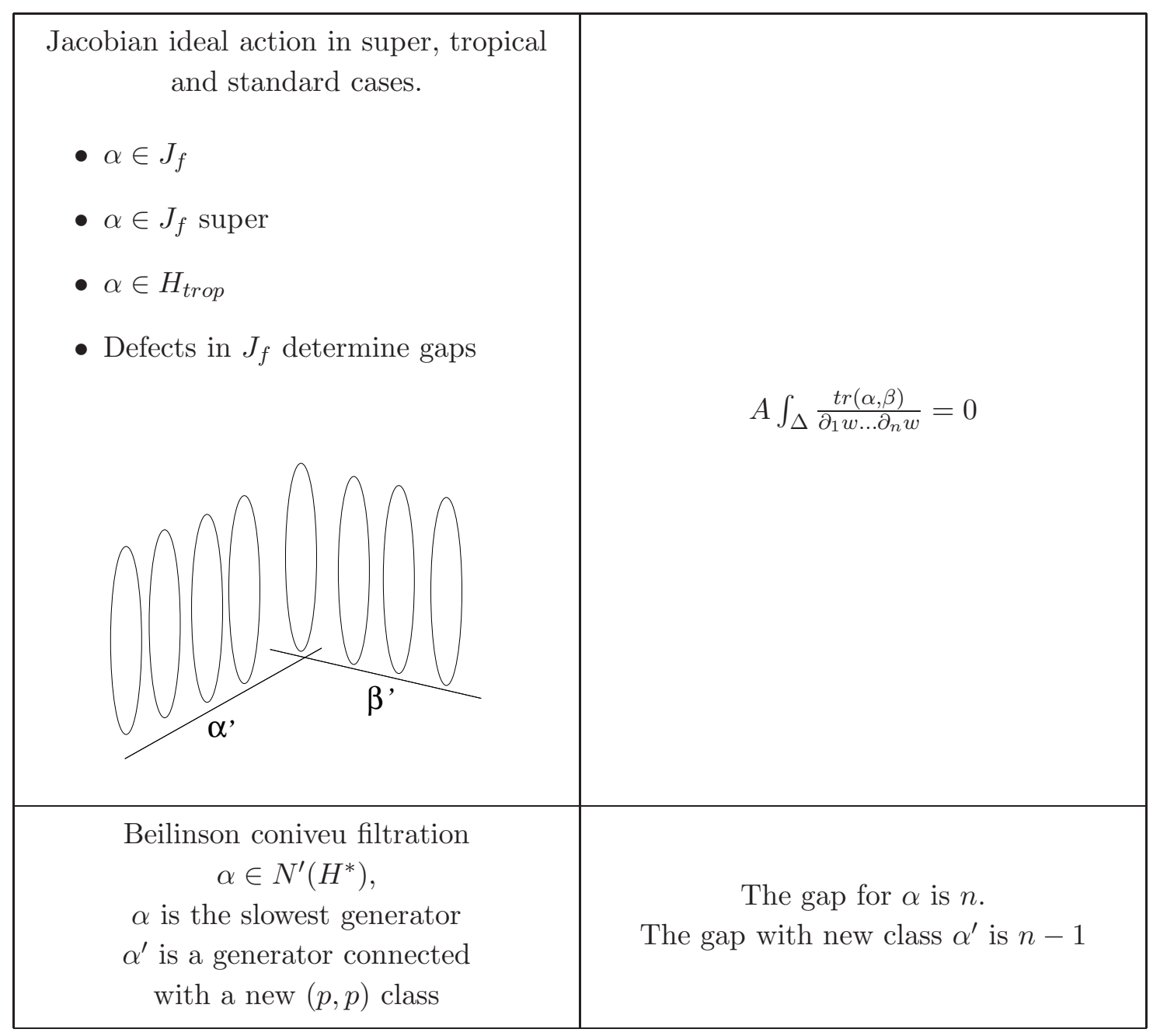

Table 2. Small gap drops for coniveau elements.

formula shows when this action is trivial. This allows us to compute $l_{G^{\prime}}(G)$ and $B\left(G^{\prime}\right)$. Based on these calculations, in the second row of the table, we connect Beilinson coniveu filtration $N^{\prime}\left(H^{*}\right)$ with relatively small gap drops.

Certain strictness properties [26] may obstruct the appearance of small gap drops and hence yield counterexamples of the Hodge conjecture. As will be discussed in [13], we may formulate the following conjecture.

Conjecture 8.4. The dimension of supercohomology is determined by Jacobian ring, gaps, and monodromy effect $M E$.

This conjecture implies that, in Example 4.1, $h^{(1,2)}\left(M^{\left(q_{1}, q_{2}\right)}\right)=83$ when $\left(q_{1}, q_{2}\right) \in$ $\{(1,5),(2,4)\}$. Thus, there are possible anomalous cycles. For a detailed explanation, see $[13,26]$. 


\section{Acknowledgements}

The authors thank V. Bouchard, C. Doran, M. Kontsevich, T. Pantev, and C. Vafa for useful discussions. R.G. and M.K. received financial support from the Austrian Research Funds (FWF) under grant numbers I192 and P21239. R.G. received additional financial support from NSERC. L.K. was funded by NSF Grant DMS0600800, NSF FRG Grant DMS-0652633, FWF Grant P20778, and an ERC Grant. A.N. received financial support from FWF Grant P20778.

\section{A A-model super CY hypersurface $\Leftrightarrow$ B-model quasihomogeneity}

In this appendix, we will prove a theorem concerning the quasihomogeneity of $\widetilde{W}$ given by (4.10) and prove a corollary concerning the $t \rightarrow-\infty$ limit.

Theorem A.1. A member of the hypersurface family

$$
\mathbf{W C P}_{\left(Q_{1}, \ldots, Q_{m} \mid q_{1}, \ldots, q_{n}\right)[s]}^{m-1 \mid n}
$$

is super Calabi-Yau if and only if

$$
\widetilde{W}=\sum_{i=1}^{m} \prod_{j=1}^{m} y_{j}^{M_{j i}}+e^{t / s} \prod_{j=1}^{m} y_{j} \prod_{b=1}^{n} x_{b}^{-1}+\sum_{a=1}^{n}\left(1+x_{a}^{-2} \hat{\eta}_{a} \hat{\gamma}_{a}\right) \prod_{b=1}^{n} x_{b}^{N_{b a}}
$$

is quasihomogeneous of some degree $s^{\prime}$ for all values of $t$.

Proof. Assume that $\widetilde{W}$ is quasihomogeneous of degree $s^{\prime}$ for all values of $t$. It follows that

$$
s^{\prime}=\sum_{j=1}^{m} n_{y_{j}} M_{j i}=\sum_{b=1}^{n} n_{x_{b}} N_{b a}=\sum_{j=1}^{m} n_{y_{j}}-\sum_{b=1}^{n} n_{x_{b}}
$$

and

$$
0=-2 n_{x_{a}}+n_{\hat{\eta}_{a}}+n_{\hat{\gamma}_{a}}
$$

where $n_{y_{j}}, n_{x_{b}}, n_{\hat{\eta}_{a}}$, and $n_{\hat{\gamma}_{a}}$ are the weights of $y_{j}, x_{b}, \hat{\eta}_{a}$, and $\hat{\gamma}_{a}$, respectively. Combining the first two equalities of (A.1) with (4.6) yields

$$
\sum_{i=1}^{m} n_{y_{i}}=\frac{s^{\prime}}{s} \sum_{i=1}^{m} Q_{i}, \quad \sum_{a=1}^{n} n_{x_{a}}=\frac{s^{\prime}}{s} \sum_{a=1}^{n} q_{a}
$$

Using (A.3) in (A.1) yields

$$
\sum_{i=1}^{m} Q_{i}-\sum_{a=1}^{n} q_{a}=s
$$

which is the super Calabi-Yau condition. 
To prove the converse, assume that the super Calabi-Yau condition holds. It is always possible to find $n_{y_{j}}$ and $n_{x_{b}}$ which satisfy the first two equalities of (A.1). Furthermore, it is always possible to satisfy (A.2). Thus, the first and third terms of $\widetilde{W}$ can always be chosen to be quasihomogeneous of some degree $s^{\prime}$. It remains to show that the second term of $\widetilde{W}$ is quasihomogeneous of degree $s^{\prime}$ for all values of $t$. Combining the first two equalities of (A.1) with (4.6) once again yields (A.3). Using (A.3) in the super Calabi-Yau condition yields

$$
\sum_{j=1}^{m} n_{y_{j}}-\sum_{b=1}^{n} n_{x_{b}}=s^{\prime}
$$

and hence the second term of $\widetilde{W}$ is quasihomogeneous of degree $s^{\prime}$ for all values of $t$.

Remark A similiar phenomenon was observed [10] when the A-model target space is a crepant resolution of

$$
\mathbf{W C P}_{\left(Q_{11}, \ldots, Q_{1 m_{1}} \mid q_{11}, \ldots, q_{1 n}\right)}^{m_{1}-1 \mid n} \times \cdots \times \mathbf{W C P}_{\left(Q_{n 1}, \ldots, Q_{n m_{n}} \mid q_{n 1}, \ldots, q_{n n}\right)}^{m_{n}-1 \mid n}
$$

Corollary A.1. Let $\widetilde{W}$ be the superpotential of the super Landau-Ginzburg orbifold which is mirror to a member $\{G=0\}$ of the hypersurface family $\mathbf{W C P}_{\left(Q_{1}, \ldots, Q_{m} \mid q_{1}, \ldots, q_{n}\right)[s]}^{m-1 \mid n}$. Then, in the limit $t \rightarrow-\infty$, the following are true:

(i) $\widetilde{W}$ is quasihomogeneous of some degree $s^{\prime}$.

(ii) The quantity

$$
\hat{c}=\sum_{i=1}^{m}\left(1-\frac{2 Q_{i}}{s}\right)-\sum_{a=1}^{n}\left(1-\frac{2 q_{a}}{s}\right)
$$

associated with $G$ is equal to the quantity

$$
\hat{\tilde{c}}=\sum_{i=1}^{m}\left(1-\frac{2 n_{y_{i}}}{s^{\prime}}\right)+\sum_{a=1}^{m}\left(1-\frac{2 n_{x_{a}}}{s^{\prime}}\right)-\sum_{a=1}^{n}\left(1-\frac{2 n_{\hat{\eta}_{a}}}{s^{\prime}}\right)-\sum_{a=1}^{n}\left(1-\frac{2 n_{\hat{\gamma}_{a}}}{s^{\prime}}\right)
$$

associated with $\widetilde{W}$.

Proof.

(i) This follows from the discussion in the second paragraph of the proof of Theorem A.1.

(ii) This follows by combining the terms in $\hat{\tilde{c}}$ which are summed over $a$ and then using (A.2) and (A.3) in turn, i.e. 


$$
\begin{aligned}
\hat{\tilde{c}} & =\sum_{i=1}^{m}\left(1-\frac{2 n_{y_{i}}}{s^{\prime}}\right)-\sum_{a=1}^{m}\left[1-\frac{2}{s^{\prime}}\left(n_{\hat{\eta}_{a}}+n_{\hat{\eta}_{a}}-n_{x_{a}}\right)\right] \\
& =\sum_{i=1}^{m}\left(1-\frac{2 n_{y_{i}}}{s^{\prime}}\right)-\sum_{a=1}^{n}\left(1-\frac{2 n_{x_{a}}}{s^{\prime}}\right) \\
& =\sum_{i=1}^{m}\left(1-\frac{2 Q_{i}}{s}\right)-\sum_{a=1}^{n}\left(1-\frac{2 q_{a}}{s}\right)=\hat{c} .
\end{aligned}
$$

\section{References}

[1] E. Witten, Phases of $N=2$ theories in two dimensions, Nucl. Phys. B403 (1993) 159 [hep-th/9301042].

[2] D. R. Morrison and M. R. Plesser, Summing the instantons: Quantum cohomology and mirror symmetry in toric varieties, Nucl. Phys. B440 (1995) 279 [hep-th/9412236].

[3] K. Hori and C. Vafa, Mirror symmetry, [hep-th/0002222].

[4] R. Schimmrigk, Critical superstring vacua from noncritical manifolds: A novel framework for string compactifications, Phys. Rev. Lett. 70 (1993) 3688 [hep-th/9210062];

R. Schimmrigk, Mirror symmetry and string vacua from a special class of Fano varieties, Int. J. Mod. Phys. A11 (1996) 3049 [hep-th/9405086].

[5] P. Candelas, E. Derrick and L. Parkes, Generalized Calabi-Yau manifolds and the mirror of a rigid manifold, Nucl. Phys. B407 (1993) 115 [hep-th/9304045].

[6] S. Sethi, Supermanifolds, rigid manifolds and mirror symmetry, Nucl. Phys. B430 (1994) 31 [hep-th/9404186].

[7] R. S. Garavuso, M. Kreuzer and A. Noll, Fano hypersurfaces and Calabi-Yau supermanifolds, JHEP 03 (2009) 007 [arXiv:0812.0097].

[8] M. Aganagic and C. Vafa, Mirror symmetry and supermanifolds, [hep-th/0403192].

[9] C. Ahn, Mirror symmetry of Calabi-Yau supermanifolds, Mod. Phys. Lett. A20 (2005) 407 [hep-th/0407009].

[10] A. Belhaj, L. B. Drissi, J. Rasmussen, E. H. Saidi and A. Sebbar, Toric Calabi-Yau supermanifolds and mirror symmetry, J. Phys. A38 (2005) 6405 [hep-th/0410291].

[11] R. Ricci, Super Calabi-Yau's and special Lagrangians, JHEP 03 (2007) 048 [hep-th/0511284].

[12] R. Ahl Laamara, A. Belhaj, L. B. Drissi and E. H. Saidi, On local Calabi-Yau supermanifolds and their mirrors, J. Phys. A39 (2006) 5965 [hep-th/0601215].

[13] R. S. Garavuso, L. Katzarkov and A. Noll, Hodge theory and supermanifolds, in preparation.

[14] A. Schwarz, Sigma-models having supermanifolds as target spaces, Lett. Math. Phys. 38 (1996) 91 [hep-th/9506070].

[15] S. Seki and K. Sugiyama, Gauged linear sigma model on supermanifold, [hep-th/0503074].

[16] C. Vafa, String vacua and orbifoldized L-G models, Mod. Phys. Lett. A4 (1989) 1169;

K. A. Intriligator and C. Vafa, Landau-Ginzburg orbifolds, Nucl. Phys. B339 (1990) 95.

[17] M. Ballard, D. Favero and L. Katzarkov, Orlov spectra: bounds and gaps, [arXiv:1012.0864]. 
[18] L. Katzarkov, Homological Mirror Symmetry and algebraic cycles, Riemannian topology and geometric structures on manifolds, K. Galicki and S. R. Simanca (eds.), Progr. Math. 271 (2009) 63, Birkhäuser, Boston.

[19] D. Orlov, Derived categories of coherent sheaves and triangulated categories of singularities, Algebra, arithmetic, and geometry: in honor of Yu. I. Manin, Vol. II, Y. Tschinkel and Y. Zarhin (eds.), Progr. Math. 270 (2009) 503, Birkhäuser, Boston [math/0503632].

[20] F. Bardelli and S. Müller-Stach, Algebraic cycles on certain Calabi-Yau threefolds, Math. Z. 215 (1994) 569.

[21] C. Voisin, The Griffiths group of a general Calabi-Yau threefold is not finitely generated, Duke Math. J. 102 (2000) 151.

[22] A. Iliev and L. Katzarkov, Griffiths Groups of Fano CY, in preparation.

[23] L. Katzarkov, M. Kontsevich and T. Pantev, Hodge theory aspects of mirror symmetry, [arXiv:0806.0107].

[24] D. Orlov, Remarks on generators and dimensions of triangulated categories, Moscow Math. J. 9 (2009) 153 [arXiv:0804.1163].

[25] R. Rouquier, Dimensions of triangulated categories, J. K-Theory 1 (2008) 193 [math/0310134].

[26] D. Favero and L. Katzarkov, Hodge theory, gaps and spectra, in preparation.

[27] A. Beilinson, A remark on primitive cycles and the Radon transform, [arXiv:1011.2446]. 